\title{
THREE RED GIANTS WITH SUBSTELLAR-MASS COMPANIONS*
}

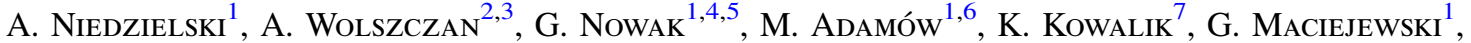 \\ B. DeKa-SZYManKIEWICZ ${ }^{1}$, AND M. AdAMCZYK ${ }^{1}$ \\ ${ }^{1}$ Toruń Centre for Astronomy, Nicolaus Copernicus University in Toruń, Grudziadzka 5, 87-100 Toruń, Poland; Andrzej.Niedzielski@umk.pl \\ ${ }^{2}$ Department of Astronomy and Astrophysics, Pennsylvania State University, 525 Davey Laboratory, University Park, PA 16802, USA; alex@astro.psu.edu \\ ${ }^{3}$ Center for Exoplanets and Habitable Worlds, Pennsylvania State University, 525 Davey Laboratory, University Park, PA 16802, USA \\ ${ }_{5}^{4}$ Instituto de Astrofísica de Canarias, C/Vía Láctea, s/n, E-38205-La Laguna (Tenerife), Spain \\ ${ }^{5}$ Departamento de Astrofísica, Universidad de La Laguna, E-38206 La Laguna, Tenerife, Spain \\ ${ }^{6}$ McDonald Observatory and Department of Astronomy, University of Texas at Austin, 2515 Speedway, Stop C1402, Austin, TX 78712-1206, USA \\ ${ }^{7}$ National Center for Supercomputing Applications, University of Illinois Urbana-Champaign, 1205 West Clark Street, MC-257, Urbana, IL 61801, USA \\ Received 2014 June 11; accepted 2015 January 27; published 2015 April 3
}

\begin{abstract}
We present three giant stars from the ongoing Penn State-Toruń Planet Search with the Hobby-Eberly Telescope, which exhibit radial velocity $(\mathrm{RV})$ variations that point to the presence of planetary-mass companions around them. $\mathrm{BD}+49828$ is a $M=1.52 \pm 0.22 M_{\odot} \mathrm{K} 0$ giant with a $m$ sin $i=1.6_{-0.2}^{+0.4} M_{J}$ minimum mass companion in $a=4.2_{-0.2}^{+0.32} \mathrm{AU}\left(2590_{-180}^{+300} \mathrm{~d}\right), e=0.35_{-0.10}^{+0.24}$ orbit. HD 95127, a $\log L / L_{\odot}=2.28 \pm 0.38, R=20 \pm 9 R_{\odot}$, $M=1.20 \pm 0.22 M_{\odot} \mathrm{K} 0$ giant, has a $m \sin i=5.01_{-0.44}^{+0.61} M_{J}$ minimum mass companion in $a=1.28_{-0.01}^{+0.01} \mathrm{AU}$ $\left(482_{-5}^{+5} \mathrm{~d}\right), e=0.11_{-0.06}^{+0.15}$ orbit. Finally, $\mathrm{HD} 216536$ is a $M=1.36 \pm 0.38 M_{\odot}$ K0 giant with a $m \sin i=1.47_{-0.12}^{+0.20} M_{J}$ minimum mass companion in $a=0.609_{-0.002}^{+0.002}$ AU $\left(148.6_{-0.7}^{+0.7} \mathrm{~d}\right), e=0.38_{-0.10}^{+0.12}$ orbit. Both HD $95127 \mathrm{~b}$ and HD $216536 \mathrm{~b}$ in their compact orbits are very close to the engulfment zone and hence prone to ingestion in the near future. BD+49 $828 \mathrm{~b}$ is among the longest-period planets detected with the RV technique until now and it will remain unaffected by stellar evolution up to a very late stage of its host. We discuss general properties of planetary systems around evolved stars and planet survivability using existing data on exoplanets in more detail.
\end{abstract}

Key words: planetary systems - stars: individual (BD+49 828 b, HD 95127 b, HD 216536 b)

\section{INTRODUCTION}

Searches for planets around stars that evolved off the main sequence (MS) represent an important ingredient of the exoplanet research. They extend the known population of planets to those orbiting massive and intermediate-mass stars. They also provide the best tool to study star-planet interactions induced by stellar evolution. Finally, these searches furnish high-quality spectroscopic data that can be used in a wide range of studies related to stellar evolution.

Intermediate-mass and massive MS stars have high effective temperatures and rotate rapidly. With only a few, rotationbroadened spectral lines, these stars are not suitable for highprecision radial velocity (RV) searches for planetary companions. Consequently, planets around intermediate-mass and massive MS stars discovered occasionally in transit searches (cf. Borucki et al. 2011; Schwamb et al. 2013) are very difficult to confirm with RV measurements. The main source of data on such planetary systems are RV searches that focus on subgiant and giant stars that are evolving off the MS, cooling down, and considerably slowing their rotation. The most massive hosts of planetary systems come almost exclusively from such surveys (e.g. Sato et al. 2007, 2010, 2012, 2013). Surveys of this kind have been uncovering a population of planetary systems that are descendants of an undetermined original sample of planets around intermediate-mass and massive stars, carrying with them imprints of the effects of several gigayears of dynamical evolution enforced by an evolving star. They have

\footnotetext{
* Based on observations obtained with the Hobby-Eberly Telescope, which is a joint project of the University of Texas at Austin, the Pennsylvania State University, Stanford University, Ludwig-Maximilians-Universität München, and Georg-August-Universität Göttingen.
}

demonstrated, for example, a paucity of planets within 0.5 AU of their parent stars (Johnson et al. 2007; Sato et al. 2008), a borderline currently broken by Kepler $91 \mathrm{~b}$, a planet at only $a / R_{\star}=2.46$ (Barclay et al. 2014; Lillo-Box et al. 2014).

The other frequently discussed effects are the higher frequency of planets around more massive stars and the stellar mass-planetary system mass relation (Lovis \& Mayor 2007; Johnson et al. 2010, 2011). Such stars also seem to prefer massive, $m_{p} \geqslant 1.5 M_{J}$, planets (Sato et al. 2007; Niedzielski et al. 2009; Bowler et al. 2010) and a more frequent occurrence of brown-dwarf-mass companions, which are deficient around solar-mass stars on the MS (Liu et al. 2008; Niedzielski et al. 2009; Mitchell et al. 2013; Niedzielski et al. 2013).

Disentangling information on the original planet population from the available data on planets around giants requires a detailed understanding of the interaction between an orbiting planet and an evolving star. In fact, the importance of the influence of tidal interactions on orbital evolution was recognized long before the first exoplanets were discovered (see Kopal 1972; Counselman 1973; Zahn 1977 and references therein). Livio (1982) studied the net effect of accretion drag and mass loss in a star-planet system. The first attempts to predict planet survival upon stellar evolution were presented by Choi (1980), Choi \& Vila (1981), Livio \& Soker (1983, 1984), and Vila (1984). Detailed effects of brown dwarf or planet capture by an evolving star were numerically modeled in Siess \& Livio (1999a, 1999b). The future of the solar system in the context of the Sun's evolution was studied by Duncan \& Lissauer (1998), Rybicki \& Denis (2001), and Schröder \& Connon Smith (2008). 
A more general picture of stellar-evolution-induced starplanet interaction, including tidal interactions, was presented in Rasio et al. (1996), Villaver \& Livio (2007), Carlberg et al. (2009), Jackson et al. (2009), and Nordhaus et al. (2010). Three possible outcomes of the orbital evolution of planets affected by tidal forces and stellar evolution, somewhat analogous to those in a planet-satellite system (Counselman 1973), were presented in Villaver \& Livio (2009). Most recent studies include effects such as the evolution of the primary's spin (Nordhaus \& Spiegel 2013) or interactions with the Galactic environment (Veras et al. 2014). The influence of stellar wind and tidal force prescriptions on planetary orbit evolution has been recently illustrated in Villaver et al. (2014).

In this paper we present three planetary-mass companions around solar-mass stars from the ongoing Penn State-Torun Planet Search (PTPS) with the Hobby-Eberly Telescope (HET; Niedzielski et al. 2007; Niedzielski \& Wolszczan 2008), BD+49 828, HD 95127, and HD 216536, and discuss their fate through their host evolution. We also summarize the available data on planetary systems of evolved stars and briefly discuss properties of planetary systems at various stages of their hosts stellar evolution.

The plan of the paper is as follows. In Section 2 we describe the observations and present our targets and their basic parameters, the measurements, and the analysis of RVs. In Section 3 we present the results of the Keplerian modeling of RV variations, while other sources of stellar variability are considered in Section 4. Finally, in Section 5 we discuss our results, the fate of planets orbiting giant stars, and some additional problems related to evolved planetary systems.

\section{OBSERVATIONS, STELLAR PARAMETERS, AND RADIAL VELOCITIY MEASUREMENTS}

\subsection{Observations}

The spectroscopic observations presented here were performed with HET (Ramsey et al. 1998) and its High Resolution Spectrograph (HRS; Tull 1998) in the queue scheduled mode (Shetrone et al. 2007). The spectrograph was used in the $R=60,000$ resolution mode with an $\mathrm{I}_{2}$ gas cell inserted into the optical path, and it was fed with a $2^{\prime \prime}$ fiber. The configuration and observing procedure employed in our program were practically identical to those described by Cochran et al. (2004).

$\mathrm{BD}+49828(\mathrm{AG}+49$ 353) is a K0 giant (Dieckvoss \& Heckmann 1997) with $B=9.43 \pm 0.03$ (Høg et al. 2000) and $V=9.38 \pm 0.02$. We collected 42 epochs of precise RVs for this star over 3134 days $(8.58 \mathrm{yr}$ ), between MJD 53225 and 56359. A typical signal-to-noise ratio $(\mathrm{S} / \mathrm{N})$ of about 200 was reached in 540-1600 s depending on atmospheric conditions.

RVs of HD 95127 (BD+44 2038), a K0 giant (Cannon \& Pickering 1918-1924) with $B=10.825 \pm 0.045$ and $V=$ $8.15 \pm 0.01$ (Høg et al. 2000) were measured at 41 epochs over the period of 2929 days ( $8 \mathrm{yr}$ ) between MJD 53024 and 55953. The $\mathrm{S} / \mathrm{Ns}$ of the combined star and iodine spectra ranged from 80 to 250 . The $\mathrm{S} / \mathrm{N}$ of the stellar template was 480 . The exposure time ranged between 170 and $800 \mathrm{~s}$, depending on the atmospheric conditions. Hipparcos parallax from van Leeuwen (2007) puts this star at a distance of $332 \pm 110 \mathrm{pc}$.

HD $216536(\mathrm{BD}+434329$ ) is a K0 giant (Cannon \& Pickering 1918-1924) with $B=10.42 \pm 0.03$ and $V=9.23 \pm$ 0.02 (Høg et al. 2000). The star was observed over 2756 days
Table 1

Stellar Parameters of Program Stars

\begin{tabular}{llll}
\hline \hline Parameter & BD+49 828 & HD 95127 & HD 216536 \\
\hline$V($ mag $)$ & $9.38 \pm 0.02$ & $8.15 \pm 0.01$ & $9.23 \pm 0.02$ \\
Spectral type & $\mathrm{K} 0$ & $\mathrm{~K} 0$ & $\mathrm{~K} 0$ \\
$\pi($ mas $)$ & $\cdots$ & $3.06 \pm 0.99$ & $\ldots$ \\
$T_{\text {eff }}(\mathrm{K})$ & $4943 \pm 30$ & $4218 \pm 69$ & $4639 \pm 45$ \\
$\log g$ & $2.85 \pm 0.09$ & $1.78 \pm 0.3$ & $2.36 \pm 0.21$ \\
{$[\mathrm{Fe} / \mathrm{H}]$} & $-0.19 \pm 0.06$ & $-0.18 \pm 0.05$ & $-0.17 \pm 0.09$ \\
$v_{\text {rot }} \sin i_{\star}\left(\mathrm{km} \mathrm{s}^{-1}\right)$ & $1.7 \pm 0.8$ & $2.6 \pm 0.7$ & $2.6 \pm 0.5$ \\
$\log L_{\star} / L_{\odot}$ & $1.47 \pm 0.13$ & $2.28 \pm 0.38$ & $1.80 \pm 0.21$ \\
$M_{\star} / M_{\odot}$ & $1.52 \pm 0.22$ & $1.20 \pm 0.22$ & $1.36 \pm 0.38$ \\
$R_{\star} / R_{\odot}$ & $7.6 \pm 1.3$ & $20 \pm 9$ & $12.5 \pm 4.0$ \\
$\log ($ Age $)(\mathrm{yr})$ & $9.37 \pm 0.18$ & $9.74 \pm 0.27$ & $9.58 \pm 0.33$ \\
$P\left(\sin i_{\star}\right)^{-1}($ days $)$ & $226 \pm 114$ & $389 \pm 204$ & $243 \pm 91$ \\
$K_{\text {osc }}\left(\mathrm{m} \mathrm{s}^{-1}\right)$ & $4.5_{-1.8}^{+3.1}$ & $37_{-25}^{+79}$ & $11_{-6}^{+15}$ \\
$P_{\text {osc }}($ days $)$ & $0.13_{-0.05}^{+0.08}$ & $1.08_{-0.81}^{+1.73}$ & $0.39_{-0.25}^{+0.56}$ \\
\hline
\end{tabular}

(7.54 yr), between MJD 53544 and 56300. The typical S/N of 250 was reached in 455-1200 s exposures depending on actual conditions.

\subsection{Stellar Parameters}

All three giants discussed here belong to the PTPS Red Giant Clump sample. They have been studied in detail by Zieliński et al. (2012), who estimated their atmospheric parameters by means of the method of Takeda et al. (2005a, 2005b) and intrinsic colors. In a more detailed chemical-abundance analysis of Adamów et al. (2014) with SME (Valenti \& Piskunov 1996), no anomalies were detected and new rotation velocities were presented.

Luminosity of HD 95127 was calculated from the Hipparcos parallax and we found it to be surprisingly high for a $\log g=3.82 \pm 0.27$ and $T_{\text {eff }}=4807 \pm 90 \mathrm{~K}$ star (Zieliński et al. 2012). To clarify that mismatch we reanalyzed all available HET/HRS spectra with no iodine imprinted and performed spectroscopic analysis with the method of Takeda et al. (2005a, 2005b) again. The resulting significantly different atmospheric parameters are presented in Table 1.

Using these parameters, the mass and age were derived with the Bayesian method based on the Jørgensen \& Lindegren (2005) formalism and modified by da Silva et al. (2006), in which we used theoretical isochrones from Bressan et al. (2012). For the other two stars, luminosity was obtained, together with the mass and age, applying the Bayesian approach described above.

Stellar radii were estimated either from the derived masses and $\log g$ values obtained from the spectroscopic analysis or using derived luminosities and effective temperatures.

With the rotational velocities from Adamów et al. (2014) and the stellar radii, masses, and luminosities derived here we have obtained estimates of maximum rotation periods, as well as periods and amplitudes of $p$-mode oscillations from the scaling relations of Kjeldsen \& Bedding (1995). All the information on targets is presented in Table 1.

The positions of the three stars discussed in the paper on the Hertzsprung-Russel Diagram (HRD) are presented in Figure 1 together with the entire PTPS sample. In this Figure we show 332 stars from Zieliński et al. (2012), 368 stars from A. Niedzielski et al. (in preparation), and 138 stars from B. Deka 


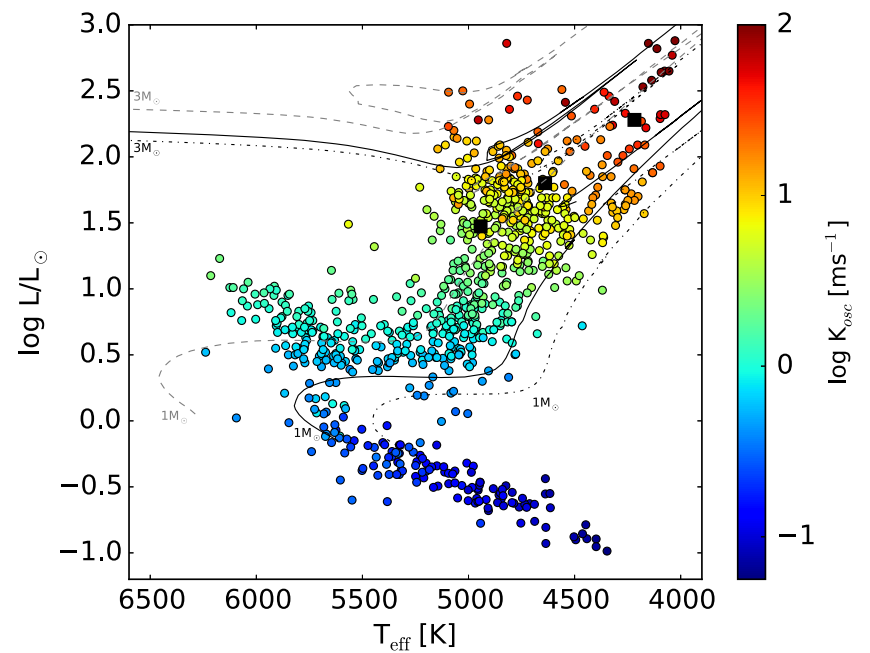

Figure 1. PTPS sample. Presented are 838 stars with detailed spectroscopic parameters available (Zieliński et al. 2012; A. Niedzielski el al., in preparation; B. Deka-SzymanKiewicz et al., in preparation). The three stars presented in this paper are marked as black rectangles. Color coded is the amplitude of pmode oscillations estimated from the scaling relations of Kjeldsen \& Bedding (1995). Evolutionary tracks of Bertelli et al. (2008) are presented for three metallicities, $[\mathrm{Fe} / \mathrm{H}]=0.0$ (solid line), -0.30 (dashed line), and 0.3 (dotted line), for illustration purposes.

et al. (in preparation) for which spectroscopically determined atmospheric parameters as well as masses, radii, and luminosities are available.

\subsection{RV Measurements}

The HRS is a general-purpose spectrograph that is neither temperature nor pressure controlled and the calibration of RV measurements with this instrument is best accomplished with the $\mathrm{I}_{2}$ cell technique. For the purpose of measuring the RVs and line bisector velocity span (BS), we have combined the method described by (Marcy \& Butler 1992; Butler et al. 1996) with that of cross-correlation used by (Queloz et al. 1995; Pepe et al. 2002). A detailed implementation of our approach is described in Nowak (2012) and Nowak et al. (2013). The typical precision of a few $\mathrm{m} \mathrm{s}^{-1}$ achieved in that way made it quite sufficient to use the Stumpff (1980) algorithm to refer the measured RVs to the solar system barycenter.

The precision of both RVs and BSs as well as the long-term stability of our RV measurements has been verified by analyzing the data derived from monitoring of stars that do not exhibit detectable RV variations and stars for which the RV or BS variations have been well established. One example of such a calibrator, discussed in Niedzielski et al. (2009), is the $\mathrm{K} 0$ giant BD+70 1068, which has $\sigma_{\mathrm{RV}}=12 \mathrm{~m} \mathrm{~s}^{-1}$ consisting of the intrinsic RV uncertainty of $7 \mathrm{~m} \mathrm{~s}^{-1}$ and the approximate $10 \mathrm{~m} \mathrm{~s}^{-1} \mathrm{RV}$ amplitude variation due to solar-type oscillations (Kjeldsen \& Bedding 1995). Nowak et al. (2013) have carried out a more detailed analysis of our RV precision and stability using the RV measurements and orbital solutions for HD 209458 and HD 88133. The precision of our BS measurements has been demonstrated for HD 166435 by the same authors.

\section{KEPLERIAN ANALYSIS}

Orbital parameters for the companions to the three stars were derived using a hybrid approach (e.g. Goździewski et al. 2003; Goździewski \& Migaszewski 2006; Goździewski et al. 2007), which combines the PIKAIA-based, global genetic algorithm (GA; Charbonneau 1995) with a faster and more precise local method. Given a periodic signal found in the RV data with the Lomb-Scargle (LS) periodogram (Lomb 1976; Scargle 1982; Press et al. 1992), we begin with launching a search for possible orbital solutions over a wide range of parameters using the GA code. The GA semi-global search usually establishes a narrow parameter range that defines the search space for the next step, in which the MPFit algorithm (Markwardt 2009) is used to find the best-fit Keplerian orbit delivered by RVLIN (Wright \& Howard 2009), modified to allow the stellar jitter to be fitted as a free parameter (Ford \& Gregory 2007; Johnson et al. 2011). The RV bootstrapping method (Murdoch et al. 1993; Kuerster et al. 1997; Marcy et al. 2005; Wright et al. 2007) is employed to assess the uncertainties of the best-fit orbital parameters defined as the width of the resulting distribution of $10^{6}$ trials of scrambled data between the $15.87 \mathrm{th}$ and 84.13th percentile.

The false alarm probability (FAP) of the final orbital solution was derived by repeating the whole hybrid analysis on $10^{5}$ sets of scrambled data.

\section{1. $B D+49828$}

The RV measurements for this star are presented in Table 2 and Figure 2. The RV variability is characterized by an amplitude of $73 \mathrm{~m} \mathrm{~s}^{-1}$ and mean uncertainty of $8.5 \mathrm{~m} \mathrm{~s}^{-1}$. The LS periodogram of these data (Figure 8) reveals only one significant peak at a period of $\sim 2410$ days at the $\mathrm{FAP}_{\mathrm{LS}}<0.01$.

The final fit of a Keplerian orbit to the data resulted in the post-fit rms residua of $11.6 \mathrm{~m} \mathrm{~s}^{-1}$ and jitter of $4.4 \mathrm{~m} \mathrm{~s}^{-1}$ in very good agreement with our estimates. The fit produced the solution with $\chi^{2}=1.35$, and, after $10^{5}$ trials, the FAP $<10^{-5}$. The final best-fit Keplerian parameters are listed in Table 5 and the model orbit is plotted together with the data in Figure 5. No additional periodic signal was detected in the post-fit RV residua (Figure 8 ).

This solution indicates the presence of a $m_{2} \sin i=$ $1.6_{-0.2}^{+0.4} M_{J}$ body in a $4.2_{-0.2}^{+0.32}$ AU, moderately eccentric, $e=0.35_{-0.10}^{+0.24}$ orbit with the period of $P=2590_{-180}^{+300}$ days. Obviously, the long orbital period of BD+49 $828 \mathrm{~b}$ is currently not very well constrained. Clearly, no less than eight years of additional observations are needed to determine it more precisely.

\section{2. $H D 95127$}

RVs of HD 95127 (Table 3 and Figure 3), with an amplitude of $404.8 \mathrm{~m} \mathrm{~s}^{-1}$ and estimated mean RV uncertainty of $5.98 \mathrm{~m} \mathrm{~s}^{-1}$, reveal a clear 483 days periodicity $\left(\mathrm{FAP}_{\mathrm{LS}}<\right.$ 0.001 ) in the corresponding LS periodogram (Figure 9).

The orbital solution includes a planet with a minimum mass of $m_{2} \sin i=5.01_{-0.44}^{+0.61} M_{J}$, in a $482_{-5}^{+5}$ day, $1.28_{-0.01}^{+0.01}$ AU, $e=0.11_{-0.06}^{+0.15}$ orbit around the star with the $\chi^{2}=1.14$ and FAP $<10^{-5}$ (Table 5, Figure 6). The post-fit residua show no significant periodicities in the corresponding LS periodogram (Figure 9).

We also note that the $47.5 \mathrm{~m} \mathrm{~s}^{-1}$ jitter and $50.9 \mathrm{~m} \mathrm{~s}^{-1}$ post-fit residua from the final model are consistent with the stellar jitter interpreted as under-sampled $p$-mode oscillations (the highly skewed distribution of jitter in our bootstraping analysis prevented a reliable uncertainty estimate). 
Table 2

Relative Radial Velocities and Bisector Span of BD+49 828

\begin{tabular}{|c|c|c|c|c|}
\hline Epoch (MJD) & $\mathrm{RV}\left(\mathrm{m} \mathrm{s}^{-1}\right)$ & $\sigma_{\mathrm{RV}}\left(\mathrm{m} \mathrm{s}^{-1}\right)$ & $\mathrm{BS}\left(\mathrm{m} \mathrm{s}^{-1}\right)$ & $\sigma_{\mathrm{BS}}\left(\mathrm{m} \mathrm{s}^{-1}\right)$ \\
\hline 53225.44499 & -20.0 & 44.1 & 79.1 & 10.8 \\
\hline 53299.22458 & -2.9 & 15.2 & -9.8 & 26.6 \\
\hline 53593.40169 & 4.0 & 14.1 & -39.1 & 24.1 \\
\hline 53608.39577 & 0.5 & 13.5 & -42.3 & 21.6 \\
\hline 53610.36711 & 15.2 & 13.6 & -38.8 & 19.5 \\
\hline 54071.31499 & 17.8 & 13.5 & -12.2 & 15.9 \\
\hline 54087.06326 & 18.9 & 13.5 & -1.4 & 16.6 \\
\hline 54101.25992 & 17.0 & 13.7 & 34.6 & 16.1 \\
\hline 54114.21772 & -5.8 & 13.4 & 10.6 & 18.4 \\
\hline 54130.16924 & 24.2 & 13.6 & 90.2 & 15.0 \\
\hline 54148.11459 & -3.4 & 13.3 & 40.7 & 17.4 \\
\hline 54449.07380 & 4.6 & 13.7 & 7.6 & 22.5 \\
\hline 54457.27139 & 22.4 & 13.8 & 13.3 & 20.3 \\
\hline 54519.10534 & 34.1 & 13.7 & 58.9 & 17.5 \\
\hline 54731.27897 & -3.5 & 14.4 & 22.9 & 23.7 \\
\hline 54756.21112 & 10.5 & 14.2 & 12.4 & 22.3 \\
\hline 54781.17038 & 19.5 & 14.6 & 34.9 & 19.1 \\
\hline 55048.43124 & -27.9 & 14.3 & 23.6 & 10.3 \\
\hline 55076.35465 & 0.2 & 15.3 & -51.9 & 10.6 \\
\hline 55079.34718 & -9.3 & 13.5 & -6.3 & 10.6 \\
\hline 55107.26189 & 8.7 & 14.0 & 15.0 & 10.7 \\
\hline 55110.26035 & 4.0 & 13.7 & 6.3 & 10.9 \\
\hline 55179.07981 & 15.4 & 14.5 & 17.9 & 22.3 \\
\hline 55206.22428 & -25.1 & 13.7 & 4.2 & 15.1 \\
\hline 55245.12414 & 2.8 & 14.6 & 74.9 & 22.4 \\
\hline 55247.11719 & -9.5 & 14.1 & 70.3 & 21.0 \\
\hline 55443.34090 & -35.4 & 15.0 & 7.6 & 32.0 \\
\hline 55478.25636 & -39.1 & 13.7 & 41.8 & 16.5 \\
\hline 55501.18840 & -26.4 & 13.8 & 39.8 & 19.6 \\
\hline 55518.16037 & -5.7 & 13.5 & 0.6 & 17.0 \\
\hline 55775.45399 & -11.8 & 13.1 & 16.5 & 16.9 \\
\hline 55791.37892 & -10.6 & 13.5 & 37.5 & 19.9 \\
\hline 55799.38224 & -18.2 & 13.1 & 10.7 & 17.8 \\
\hline 55825.30463 & -9.6 & 13.6 & -3.5 & 15.6 \\
\hline 55859.21333 & 2.3 & 13.7 & 14.8 & 13.2 \\
\hline 55931.23168 & -10.6 & 13.6 & 31.3 & 13.7 \\
\hline 55973.12528 & 5.1 & 13.3 & 40.2 & 15.8 \\
\hline 56214.24030 & 7.0 & 13.6 & 7.7 & 20.6 \\
\hline 56257.13616 & 3.9 & 13.6 & 17.7 & 15.1 \\
\hline 56324.16275 & 31.9 & 18.5 & -35.1 & 51.9 \\
\hline 56347.08323 & 4.5 & 13.6 & 54.5 & 18.0 \\
\hline 56359.08653 & 7.8 & 14.2 & 46.3 & 23.6 \\
\hline
\end{tabular}

\section{3. $H D 216536$}

The RVs for HD 216536 are presented in Table 4 and plotted in Figure 4. In this case, the mean uncertainty and amplitude characterizing the RV variability are 6.4 and $144 \mathrm{~m} \mathrm{~s}^{-1}$, respectively. The LS periodogram of the data (Figure 10) shows a single significant period of 148 days with the FAP $_{\text {LS }}<0.001$.

The hybrid modeling of these data resulted in a Keplerian fit with the post-fit rms residua of $23 \mathrm{~m} \mathrm{~s}^{-1}$ and a jitter of $17.9 \mathrm{~m} \mathrm{~s}^{-1}$ (again presenting a highly skewed distribution), consistent with our estimates of the amplitude of unresolved $p$ mode oscillations based on stellar mass and luminosity. The final, best-fit model $\left(\chi^{2}=1.29, \mathrm{FAP}<10^{-5}\right)$ parameters are listed in Table 5 and the model RV curve is plotted in Figure 7. No other periodic signal is present in the RV residua.

A minimum mass of the companion to HD 216536 is $m_{2} \sin i=1.47_{-0.12}^{+0.20} M_{J}$, and its close-in, $0.609_{-0.002}^{+0.002} \mathrm{AU}$,

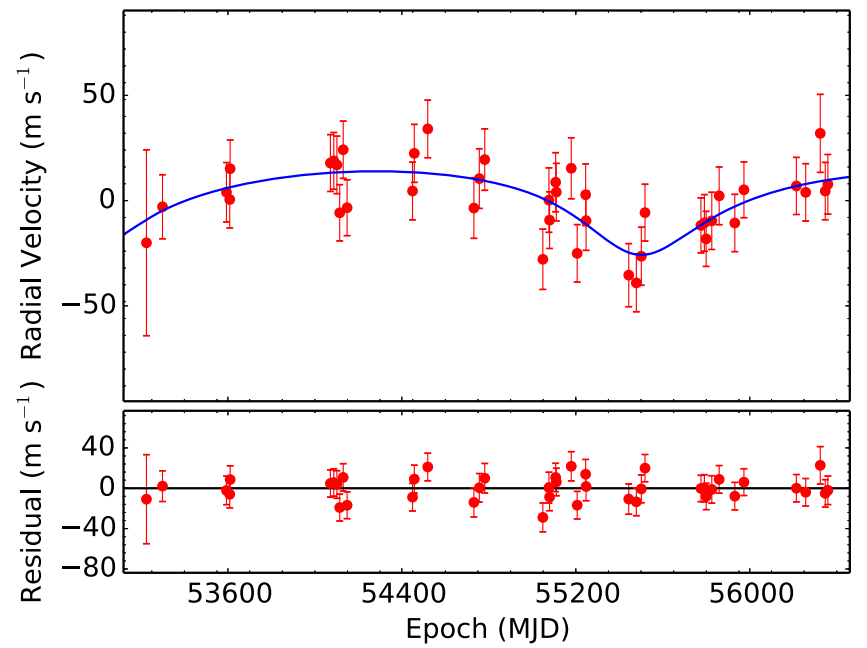

Figure 2. Radial velocities of BD+49 828 as in Table 2 plotted as a function of time together with the solution detailed in Table 5.

Table 3

Relative Radial Velocities and Bisector Span of HD 95127

\begin{tabular}{|c|c|c|c|c|}
\hline Epoch (MJD) & $\mathrm{RV}\left(\mathrm{m} \mathrm{s}^{-1}\right)$ & $\sigma_{\mathrm{RV}}\left(\mathrm{m} \mathrm{s}^{-1}\right)$ & $\mathrm{BS}\left(\mathrm{m} \mathrm{s}^{-1}\right)$ & $\sigma_{\mathrm{BS}}\left(\mathrm{m} \mathrm{s}^{-1}\right)$ \\
\hline 53024.52304 & 38.3 & 7.1 & 23.1 & 17.8 \\
\hline 53370.35059 & -82.1 & 9.1 & 12.3 & 24.0 \\
\hline 53867.22760 & -138.3 & 5.5 & 34.6 & 13.3 \\
\hline 54048.49149 & 47.8 & 5.7 & 5.4 & 18.4 \\
\hline 54129.28005 & 137.1 & 6.6 & 19.8 & 17.9 \\
\hline 54153.43199 & 120.2 & 6.1 & -4.5 & 16.9 \\
\hline 54165.40075 & 32.2 & 5.7 & 58.9 & 15.8 \\
\hline 54198.31076 & 37.0 & 4.3 & 32.6 & 13.3 \\
\hline 54212.26155 & 91.8 & 5.9 & 16.1 & 19.7 \\
\hline 54214.26450 & 87.1 & 4.8 & 2.9 & 18.3 \\
\hline 54233.22538 & -35.9 & 4.6 & 21.3 & 14.0 \\
\hline 54249.17289 & -43.3 & 5.2 & 6.8 & 15.9 \\
\hline 54249.17781 & -46.4 & 4.3 & -8.3 & 12.1 \\
\hline 54413.47777 & -88.4 & 5.3 & 55.3 & 17.1 \\
\hline 54439.43738 & -51.3 & 6.4 & 57.8 & 18.7 \\
\hline 54498.47970 & 11.5 & 7.1 & 8.8 & 21.1 \\
\hline 54532.18166 & 107.8 & 7.1 & 55.3 & 18.4 \\
\hline 54567.33124 & 150.4 & 6.9 & 22.1 & 18.5 \\
\hline 54568.09186 & 99.1 & 6.8 & 63.9 & 18.5 \\
\hline 54580.27156 & 75.8 & 5.8 & 31.9 & 15.6 \\
\hline 54618.14668 & 112.7 & 6.4 & 41.6 & 17.5 \\
\hline 54811.41721 & -40.9 & 6.4 & -19.2 & 19.5 \\
\hline 54842.33315 & -35.1 & 5.9 & 17.0 & 18.4 \\
\hline 54867.24900 & -34.6 & 6.1 & 44.6 & 18.0 \\
\hline 55173.39559 & -78.0 & 5.7 & 23.6 & 16.2 \\
\hline 55197.34812 & -4.3 & 4.8 & 13.8 & 13.3 \\
\hline 55221.51289 & -108.7 & 4.3 & -3.2 & 14.6 \\
\hline 55245.45360 & -216.0 & 6.4 & -3.8 & 14.8 \\
\hline 55358.13133 & -182.0 & 5.4 & 29.3 & 13.3 \\
\hline 55512.49483 & 56.7 & 6.7 & 46.8 & 17.5 \\
\hline 55544.40921 & 188.8 & 6.8 & 30.3 & 17.6 \\
\hline 55574.31534 & 59.4 & 5.4 & 38.6 & 13.4 \\
\hline 55577.32536 & 64.5 & 6.6 & 18.5 & 18.7 \\
\hline 55607.45310 & 89.3 & 6.3 & 23.7 & 15.0 \\
\hline 55637.15901 & 37.6 & 5.9 & 65.4 & 16.1 \\
\hline 55652.10131 & 56.9 & 4.9 & 45.4 & 11.6 \\
\hline 55691.21517 & -24.8 & 6.0 & -0.7 & 12.8 \\
\hline 55718.16090 & -89.4 & 6.1 & 0.4 & 14.1 \\
\hline 55870.50090 & -141.0 & 8.5 & 1.5 & 28.7 \\
\hline 55917.38294 & 3.1 & 5.2 & -17.7 & 13.3 \\
\hline 55953.51961 & -42.6 & 4.9 & 33.6 & 12.8 \\
\hline
\end{tabular}




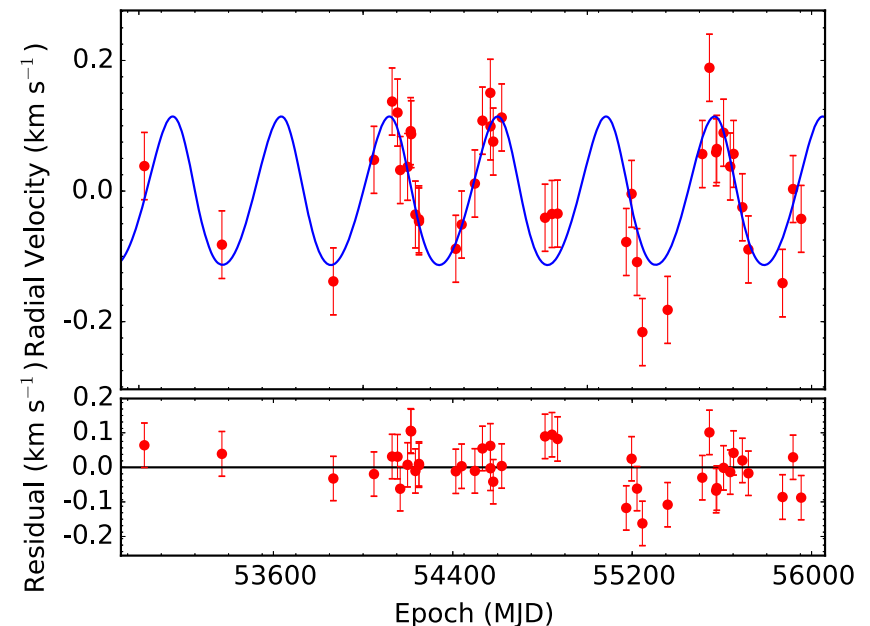

Figure 3. Radial velocities of HD 95127 as in Table 4 plotted as a function of time together with the solution detailed in Table 5 .

$P=148.6_{-0.7}^{+0.7}$ days, orbit has a moderate eccentricity, $e=0.38_{-0.10}^{+0.12}$.

\section{STELLAR ACTIVITY ANALYSIS: LINE BISECTORS, $\mathrm{H} \alpha$ VARIATIONS, AND PHOTOMETRY}

Both RV searches for planets and studies of star-planet interaction are affected by the intrinsic variability of host stars causing RV or BS variations not related to stellar reflex motion caused by the presence of an orbiting planet. Changes in spectral line shapes arising from motions in the stellar atmosphere, related to nonradial pulsations or inhomogeneous convection and/or spots combined with rotation can mimic low-level, long-period RV variations. The significant variability of red giants has been noted already by PayneGaposchkin (1954) and Walker et al. (1989) and made the nature of these variations a topic of numerous studies.

Hatzes \& Cochran (1993) showed that the low-amplitude, long-period RV variations may be attributed to pulsations, stellar activity - a spot rotating with a star, or low-mass companions with the presence of short-period, possibly $p$-mode oscillations as demonstrated in Hatzes \& Cochran (1994).

The solar-type, $p$-mode oscillations are easily observable in high-precision, photometric time-series measurements and they have been intensely studied based on CoRoT (Baglin et al. 2006) and Kepler (Gilliland et al. 2010) data, leading up to precise stellar mass determinations (de Ridder et al. 2009; Bedding et al. 2010; Kallinger et al. 2010; Hekker et al. 2011). With the typical timescales for giants, ranging from hours to days, such oscillations typically remain unresolved in lowcadence observations focused on long-term RV variations and they contribute as an additional uncertainty to the RV measurements.

Long-period RV variations in some giants have been successfully demonstrated to be due to the presence of lowmass companions (Campbell et al. 1988; Hatzes \& Cochran 1993; Vogt et al. 2000; Butler et al. 2001; Frink et al. 2002; Hatzes et al. 2003, 2006). According to the http://exoplanets.eu website, 55 giant stars with substellar-mass companions have been identified so far. However, in some giants, the nature of the observed RV variations indicating the presence of longterm, secondary periods (Payne-Gaposchkin 1954; Houk 1963) remains a riddle (Nicholls et al. 2009).
Table 4

Relative Radial Velocities and Bisector Span of HD 216536

\begin{tabular}{|c|c|c|c|c|}
\hline Epoch (MJD) & $\mathrm{RV}\left(\mathrm{m} \mathrm{s}^{-1}\right)$ & $\sigma_{\mathrm{RV}}\left(\mathrm{m} \mathrm{s}^{-1}\right)$ & $\mathrm{BS}\left(\mathrm{m} \mathrm{s}^{-1}\right)$ & $\sigma_{\mathrm{BS}}\left(\mathrm{m} \mathrm{s}^{-1}\right)$ \\
\hline 53544.38601 & -43.1 & 24.5 & -39.4 & 17.4 \\
\hline 54365.34050 & 24.9 & 23.7 & 64.5 & 14.5 \\
\hline 54658.32385 & 32.8 & 25.1 & 45.0 & 30.8 \\
\hline 55370.39329 & 53.7 & 23.6 & -5.7 & 15.4 \\
\hline 55470.35085 & -63.9 & 23.8 & -12.0 & 13.1 \\
\hline 55515.18324 & -44.2 & 24.6 & -41.7 & 12.4 \\
\hline 55518.18400 & -30.3 & 24.0 & 8.9 & 14.9 \\
\hline 55728.36984 & 8.8 & 24.0 & 26.5 & 17.3 \\
\hline 55733.37812 & 41.2 & 23.7 & 27.8 & 13.5 \\
\hline 55740.37428 & 4.0 & 24.3 & -5.8 & 17.8 \\
\hline 55753.32927 & -13.2 & 23.8 & 13.9 & 14.2 \\
\hline 55758.31630 & -25.3 & 23.9 & -4.8 & 16.5 \\
\hline 55763.29701 & -21.3 & 24.2 & 6.2 & 15.0 \\
\hline 55768.28240 & -54.0 & 23.8 & 20.3 & 13.1 \\
\hline 55775.27116 & -53.2 & 23.7 & 19.6 & 11.5 \\
\hline 55780.22843 & -68.7 & 23.9 & 5.6 & 16.9 \\
\hline 55793.43019 & -57.6 & 23.8 & 34.3 & 14.8 \\
\hline 55801.20164 & -25.2 & 23.8 & -2.5 & 13.3 \\
\hline 55810.16312 & -21.8 & 23.8 & -15.7 & 13.9 \\
\hline 55816.12715 & -40.3 & 24.2 & 1.0 & 17.9 \\
\hline 55817.38332 & -8.9 & 23.8 & -11.3 & 14.9 \\
\hline 55822.36050 & 4.5 & 23.7 & 27.5 & 13.7 \\
\hline 55825.14133 & 34.6 & 23.9 & 33.1 & 14.6 \\
\hline 55826.14602 & 49.4 & 23.8 & 29.2 & 12.4 \\
\hline 55829.10961 & 58.6 & 23.7 & -13.9 & 13.6 \\
\hline 55829.34425 & 65.0 & 23.7 & -7.1 & 12.1 \\
\hline 55830.11034 & 75.2 & 23.7 & -3.2 & 13.3 \\
\hline 55840.31604 & 66.9 & 23.8 & 42.7 & 11.3 \\
\hline 55849.31763 & 5.4 & 23.9 & 25.4 & 11.8 \\
\hline 55858.05332 & 38.7 & 23.9 & 46.9 & 12.3 \\
\hline 55888.21126 & -23.4 & 23.7 & 41.2 & 12.1 \\
\hline 55903.14193 & -44.3 & 24.0 & 17.1 & 17.8 \\
\hline 55924.08639 & -5.2 & 23.9 & 9.7 & 14.1 \\
\hline 55932.06380 & -49.4 & 23.7 & 15.1 & 10.8 \\
\hline 56073.43822 & -42.4 & 23.8 & 34.7 & 17.5 \\
\hline 56080.43409 & -21.2 & 23.7 & 16.6 & 12.4 \\
\hline 56179.39309 & 25.8 & 23.8 & 23.8 & 16.0 \\
\hline 56196.11293 & 11.0 & 23.7 & 7.5 & 13.4 \\
\hline 56210.06818 & -64.3 & 23.7 & 4.5 & 11.7 \\
\hline 56228.25215 & -56.5 & 23.9 & -7.7 & 11.8 \\
\hline 56261.14362 & 13.6 & 23.6 & 17.5 & 11.6 \\
\hline 56262.16696 & 34.9 & 23.6 & 12.1 & 12.9 \\
\hline 56282.10282 & 28.3 & 23.9 & 25.6 & 15.9 \\
\hline 56287.10367 & 4.6 & 24.3 & 40.7 & 21.4 \\
\hline 56288.09887 & 12.8 & 23.8 & 35.0 & 11.6 \\
\hline 56291.08204 & 44.2 & 23.8 & 17.2 & 12.4 \\
\hline 56300.06651 & 38.7 & 23.8 & 31.8 & 13.2 \\
\hline
\end{tabular}

Therefore, a reliable planet detection requires all the known sources of the observed RV variations to be investigated in considerable detail. We note, however, that in the case of the three stars discussed in this paper, substantial eccentricities of the best-fit Keplerian orbits alone make stellar-pulsation-based alternative explanations less likely.

Ca II H\&K resonance lines, if measurable, are good indicators of stellar activity. Unfortunately, in the HET/HRS spectra of red giants these lines are too weak to be usable. Therefore, we have developed a cross-correlation technique to monitor stellar activity with BS measurements derived from exactly the same spectra that have been used for RV determinations (Nowak 2012; Nowak et al. 2013). 


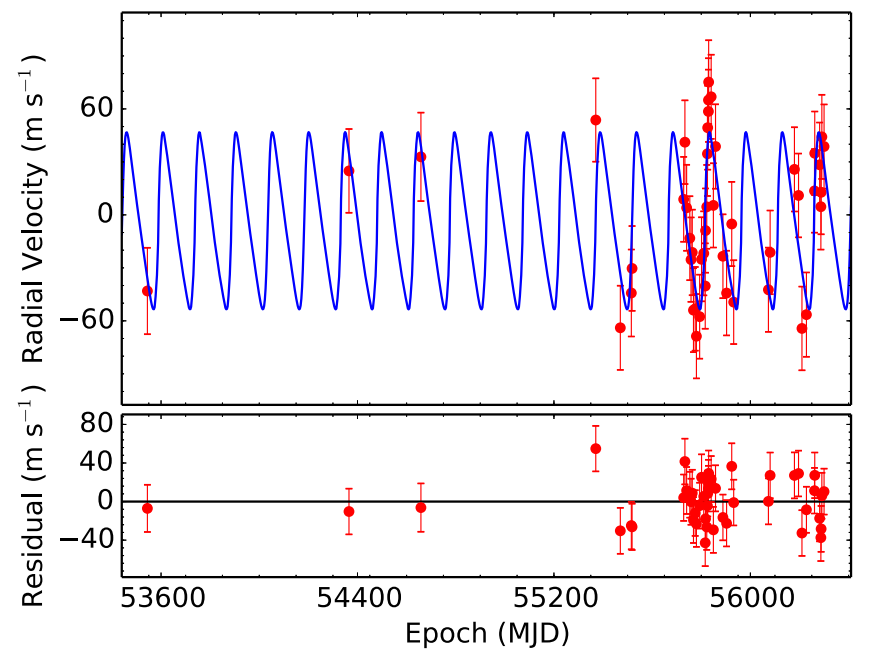

Figure 4. Radial velocities of HD 216536 as in Table 4 plotted as a function of time together with the solution detailed in Table 5.

Table 5

Orbital Parameters of BD+49 828 b, HD 95127 b, and HD 216536 b

\begin{tabular}{lccc}
\hline \hline & & & $\mathrm{HD}$ \\
Parameter & $\mathrm{BD}+49828 \mathrm{~b}$ & $\mathrm{HD} 95127 \mathrm{~b}$ & $216536 \mathrm{~b}$ \\
\hline$p(\mathrm{~d})$ & $2590_{-180}^{+300}$ & $482_{-5}^{+5}$ & $148.6_{-0.7}^{+0.7}$ \\
$T_{0}(\mathrm{MJD})$ & $55470_{-170}^{+200}$ & $53200_{-50}^{+50}$ & $53587_{-11}^{+11}$ \\
$K\left(\mathrm{~m} \mathrm{~s}^{-1}\right)$ & $18.8_{-2.0}^{+6.2}$ & $116_{-9}^{+16}$ & $50_{-4}^{+8}$ \\
$e$ & $0.35_{-0.10}^{+0.24}$ & $0.11_{-0.06}^{+0.15}$ & $0.38_{-0.10}^{+0.12}$ \\
$\omega(\mathrm{deg})$ & $170_{-30}^{+32}$ & $40_{-40}^{+37}$ & $270_{-20}^{+21}$ \\
$m_{2} \sin i\left(\right.$ closeup $\left.\mathrm{M}_{J}\right)$ & $1.6_{-0.2}^{+0.4}$ & $5.01_{-0.44}^{+0.61}$ & $1.47_{-0.12}^{+0.20}$ \\
$a(\mathrm{AU})$ & $4.2_{-0.2}^{+0.32}$ & $1.28_{-0.01}^{+0.01}$ & $0.609_{-0.002}^{+0.002}$ \\
$V_{0}\left(\mathrm{~m} \mathrm{~s}^{-1}\right)$ & $1.1_{-0.5}^{+1.4}$ & $-10.5_{-2.3}^{+3.1}$ & $-4.9_{-2.1}^{+0.7}$ \\
$\sqrt{\chi_{\nu}^{2}}$ & 1.35 & 1.14 & 1.29 \\
$\sigma_{\mathrm{RV}}\left(\mathrm{m} \mathrm{s}^{-1}\right)$ & 11.6 & 50.9 & 23.0 \\
jitter $\left(\mathrm{m} \mathrm{s}^{-1}\right)$ & $4.44_{-1.00}^{+0.35}$ & 47.5 & 17.9 \\
$N_{\mathrm{obs}}$ & 42 & 41 & 47 \\
\hline
\end{tabular}

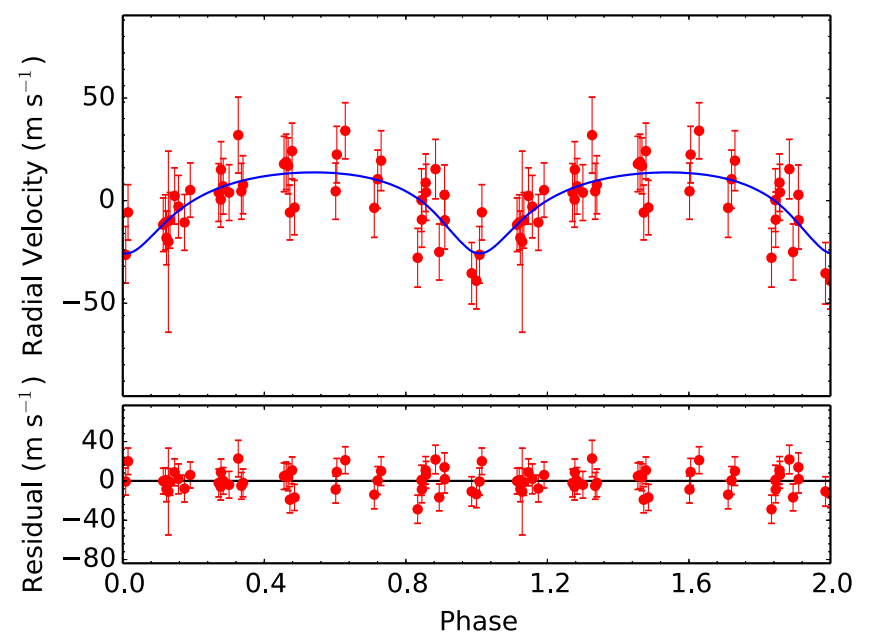

Figure 5. Radial velocities of BD+49 828 plotted as a function of orbital phase together with the solution detailed in Table 5 .

As an independent stellar activity indicator, we also measured the $\mathrm{H} \alpha$ index $\left(I_{\mathrm{H} \alpha}\right)$ from HET/HRS red spectra based on the approach presented by Gomes da Silva et al. (2012) and Robertson et al. (2013, and references therein),

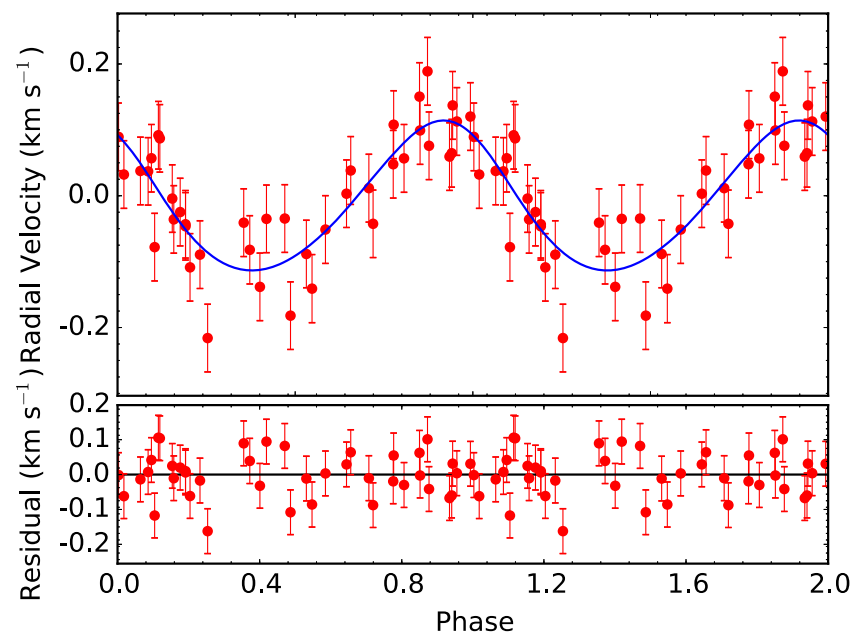

Figure 6. Radial velocities of HD 95127 plotted as a function of orbital phase together with the solution detailed in Table 5 .

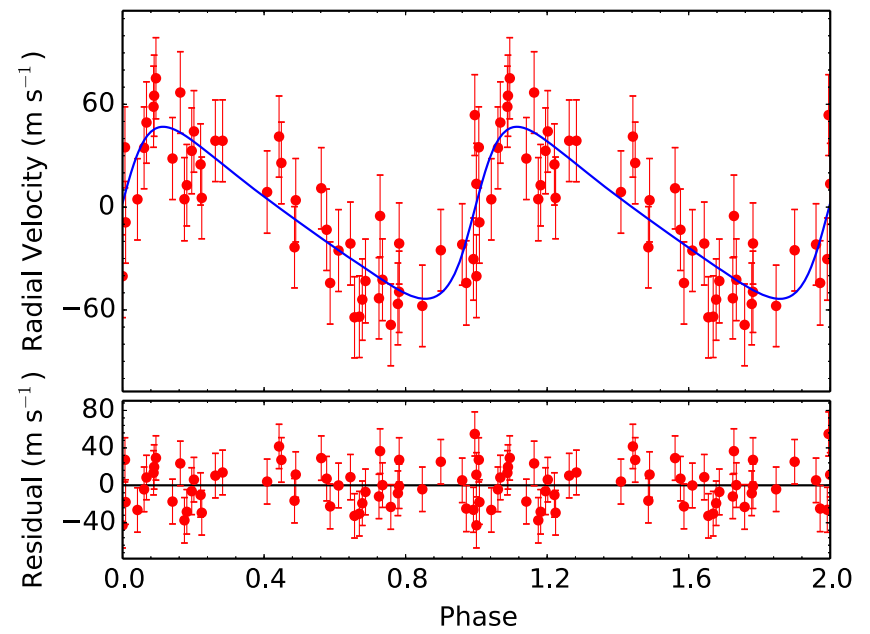

Figure 7. Radial velocities of HD 216536 plotted as a function of orbital phase together with the solution detailed in Table 5.

following the procedure described in detail in Maciejewski et al. (2013).

Both activity indicators, BS and $I_{\mathrm{H} \alpha}$, were computed at the same epochs and from the same spectra as the RVs, but such subtle effects as instrumental profile variations were not accounted for in these measurements. Consequently, they are, to some extend, subject to instrumental effects presumably not present in the RV measurements. As the $H \alpha$ line is also rather weak in $\mathrm{K}$ giants, it is especially prone to instrumental effects. To monitor such effects we also measured the index of the Fe I $654.6239 \mathrm{~nm}$ line $\left(I_{\mathrm{Fe}}\right)$, which is insensitive to stellar activity.

Also, the PTPS stars are relatively bright, which makes their photometric measurements available from the published surveys. These three kinds of data provide most of the information that we use to diagnose any possible activity of our target stars that may mimic RV variations due to the Keplerian motion.

\section{1. $B D+49828$}

The maximum rotation period of $\mathrm{BD}+49828$, given the estimated projected rotation velocity of $v_{\text {rot }} \sin i_{\star}=$ $1.7 \pm 0.8 \mathrm{~km} \mathrm{~s}^{-1}$ and stellar radius of $7.6 \pm 1.3 R_{\odot}$, is $226 \pm$ 

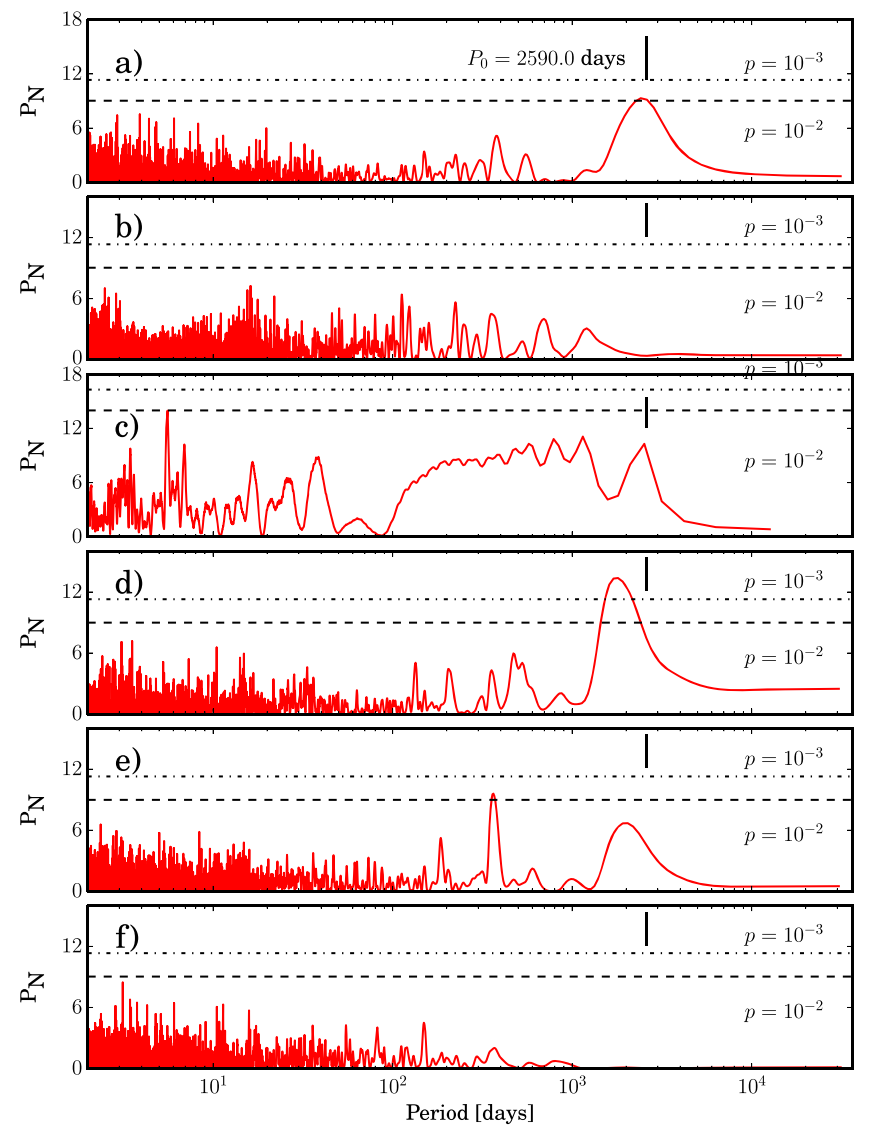

Figure 8. Lomb-Scargle periodograms for BD+49 828. From top to bottom: HET RV data, BS, WASP photometry (Butters et al. 2010), $I_{\mathrm{H} \alpha}, I_{\mathrm{Fe}}$, and RV post-fit residua. The low-signal peak in the periodogram for WASP photometry is most certainly an artifact as the available data cover only a fraction of the orbital period present in the RV data-see Section 4.1 for discussion.

114 days, much less than the orbital period, which makes rotation of a spot on the stellar surface an unlikely scenario.

There are two archival photometric time series available for this star. The Northern Sky Variablity Survey (NSVS; Woźniak et al. 2004) contains a set of 81 epochs of photometric measurements made over 216 days between MJD 51414 and 51630, which give the mean brightness and rms scatter of $9.23 \mathrm{mag}$ and $0.028 \mathrm{mag}$, respectively. The Wide Angle Search for Planets (WASP; Butters et al. 2010) archive contains a much larger data set of 5557 epochs of photometric observations made over 1262 days between MJD 53196 and 54458. These measurements are partially contemporaneous with our RV measurements and are characterized by the respective mean brightness and the rms scatter of 9.659 mag and $0.019 \mathrm{mag}$. None of these data series contain a periodic signal close to the best-fit Keplerian orbital period. The LS periodogram of the WASP observations is shown in Figure 8. An absence of any periodic signal in the existing photometric time series suggests that pulsations or a spot rotating with the star cannot be the source of the observed RV variations. However, the time covered by photometric observations is short compared to orbital period. Therefore we conservatively assume that all the observed photometric scatter originates from a spot of a size of $f=1.9 \%$ of the rotating stellar surface. Then, according to Hatzes (2002), we might expect a periodic variation of the RVs at a full amplitude of $64 \mathrm{~m} \mathrm{~s}^{-1}$. This would be similar to the observed RV variations and it would generate
BS variations at the amplitude of $45 \mathrm{~m} \mathrm{~s}^{-1}$ correlated with RV. To resolve such a situation one needs to carry out a detailed study of BSs and search their variations for periodicities that would correlate with those observed in the RV measurements.

The BS measurements for this star have a mean value of $18 \pm 33 \mathrm{~m} \mathrm{~s}^{-1}$ and amplitude of $142 \mathrm{~m} \mathrm{~s}^{-1}$ with the mean uncertainty of $19 \mathrm{~m} \mathrm{~s}^{-1}$. The Parsons correlation coefficient between the RV and the BS variations amounts to -0.09 indicating that they are independent. In addition, the BS variations do not exhibit any measurable periodicities (the LS periodogram of the $\mathrm{BS}$ variations is shown in Figure 8.) Finally, the $2590_{-180}^{+300}$ days orbital period of BD+49 $282 \mathrm{~b}$ is much too long to be related to the observed rotational period of the star, which is more than 11 times shorter than that.

Our $I_{\mathrm{H} \alpha}$ measurements for BD+49 828 present a mean value of 0.0365 and range between 0.0381 and 0.0353 , i.e., explicit variations of $7.5 \%$. At the same time $I_{\mathrm{Fe}}$ has a mean value of 0.01147 and ranges from 0.01169 to 0.01132 , i.e., varies by $3.3 \%$ accounting for nearly half of the observed $I_{\mathrm{H} \alpha}$ variations. The LS periodograms of both indices are shown in Figure 8. The $I_{\mathrm{H} \alpha}$ shows a significant periodicity of about 1750 days $(\sim 5 \mathrm{yr})$ and $I_{\mathrm{Fe}}$ data show a signficant period of about $1 \mathrm{yr}$, apparently of seasonal origin. The long-period signal is well separated from the RV period of $2590_{-180}^{+300}$ days. The $1 \mathrm{yr}$ signal is, however, close to the estimated rotation period of the star. Both LS of line profile indices show a similar pattern suggesting strong influence of instrumental effects enhanced in the case of $I_{\mathrm{H} \alpha}$ by stronger $\mathrm{S} / \mathrm{N}$. Indeed, the Parson's correlation coefficient between RV and $I_{\mathrm{H} \alpha}$ is $r=-0.344$ only while the correlation between $I_{\mathrm{H} \alpha}$ and $I_{\mathrm{Fe}}$ is $r=0.620$, far above the critical value of 0.408 (confidence level of 0.01 ). We may safely assume therefore that the observed $I_{\mathrm{H} \alpha}$ variability is induced by instrumental effects and carries no evidence of stellar activity.

Based on the discussion above, we conclude that the RV variations in BD+49 828 cannot be explained in terms of rotation induced stellar activity or pulsations. Consequently, a Keplerian modeling of the measured RV variations of the star is justified and the presence of an orbiting low-mass companion represents the most natural interpretation of the data. The observed BS variations must originate from another source that is either random or not resolved with our observations. The origin of $I_{\mathrm{H} \alpha}$ and $I_{\mathrm{Fe}}$ variations seems to be instrumental, but its nature is not clear.

\section{2. $H D 95127$}

With the estimated projected rotation velocity of $v_{\text {rot }} \sin i_{\star}=2.6 \pm 0.7 \mathrm{~km} \mathrm{~s}^{-1}$ and radius of $20 \pm 9 R_{\odot}$ the maximum rotation period of HD 95127 is $389 \pm 204$ days, much less than the orbital period.

If the observed RV variations were indeed a result of a spot rotating with the star, the observer RV semi-amplitude would require a spot of $9 \%$ of the stellar surface (Hatzes 2002). Such a spot would be easily detectable with moderate-quality photometry. The only existing extensive $V$-band photometric monitoring for HD 95127 comes from SWASP (Pollacco et al. 2006) where 540 epochs are available over only about 100 days between MJD 54091.1 and 54194.9. The mean brightness of the star was $8.394 \pm 0.012 \mathrm{mag}$ and the data show an amplitude of 0.033 mag only, three times less than expected. Ninety-five Hipparcos (ESA 1997) $H_{\mathrm{p}}$ measurements taken over 3 yr between MJD 47877 and 48961 also show a constant 

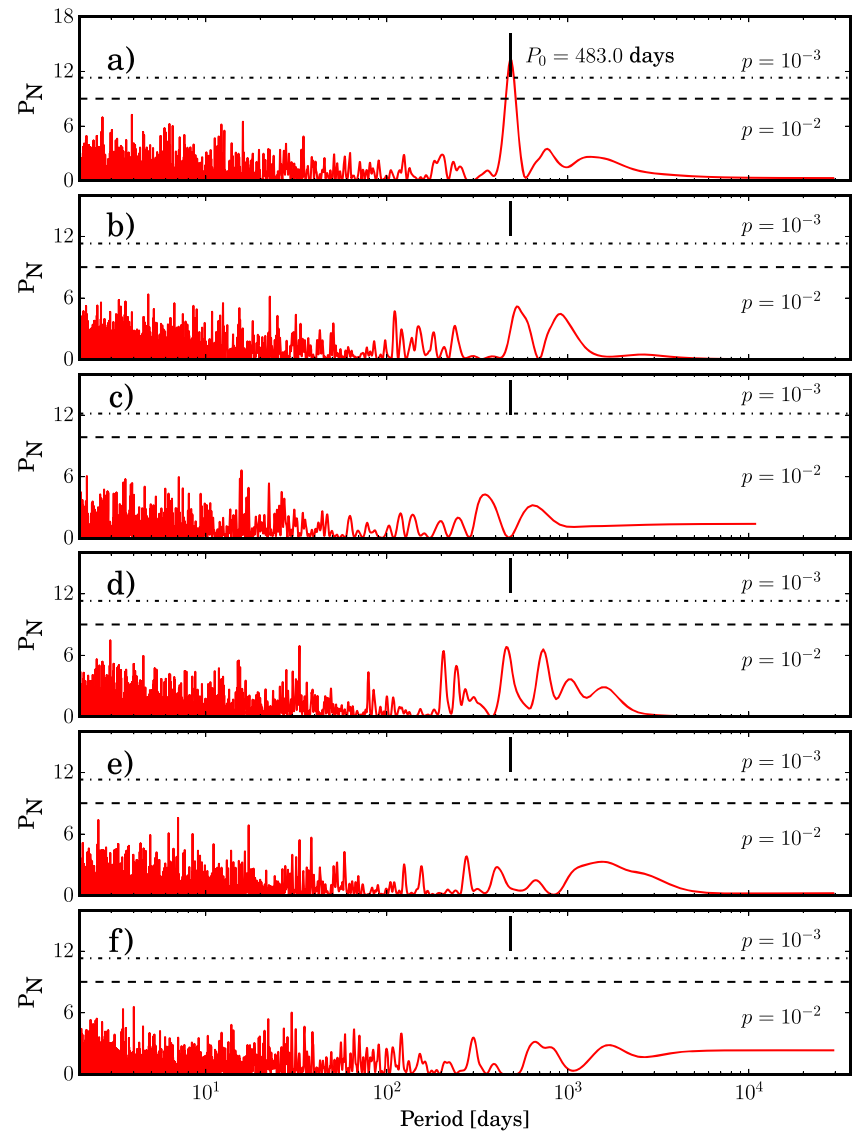

Figure 9. Lomb-Scargle periodograms for HD 95127. From top to bottom: HET RV data, BS, Hipparcos photometry (ESA 1997), $I_{\mathrm{H} \alpha}, I_{\mathrm{Fe}}$, and RV postfit residua.

brightness of $H_{p}=8.307 \pm 0.014 \mathrm{mag}$ and no significant periodicity (Figure 9). We can conclude therefore that the existing photometric data do not support the spot hypothesis. The lack of any significant periodic signals in photometric data excludes also pulsations as a possible source of RV variations.

The spot hypothesis predicts BS variations of semiamplitude of $23.7 \mathrm{~m} \mathrm{~s}^{-1}$ (Hatzes 2002) while the observed BS semi-amplitude is $42 \mathrm{~m} \mathrm{~s}^{-1}$. However, the uncertainties in BS are much larger than in RV (16.6 $\mathrm{m} \mathrm{s}^{-1}$ on average) and the mean value of BS is $23 \pm 23 \mathrm{~m} \mathrm{~s}^{-1}$. The LS analysis of BS data shows no trace of periodicity at the rotation period or at the Keplerian best-fit period (Figure 9). Moreover, the Parson's correlation coefficient between RV and BS is $r=0.25$ with the critical value of 0.4 , and no relation between these quantities can be stated. Again, the BS data do not support the spot hypothesis.

The $I_{\mathrm{H} \alpha}$ index for HD 95127 has a mean value of 0.0339 , and varies by $8.5 \%$. At the same time $I_{\mathrm{Fe}}$ index presents a mean value of 0.00983 and varies only by $1.9 \%$. None of indices shows any significant periodic signal (Figure 9). Also the correlation analysis carries no useful information on activity as both correlations between RV and $I_{\mathrm{H} \alpha}$ and between $I_{\mathrm{H} \alpha}$ and $I_{\mathrm{Fe}}$, $r=0.419$ and $r=0.424$, respectively, are at the level of critical value $(0.403)$.

Therefore, we conclude that the only significant periodic signal present in the RV data is due to an orbiting planet and there is no indication of pulsations or spot rotating with the star that can be inferred from our data.
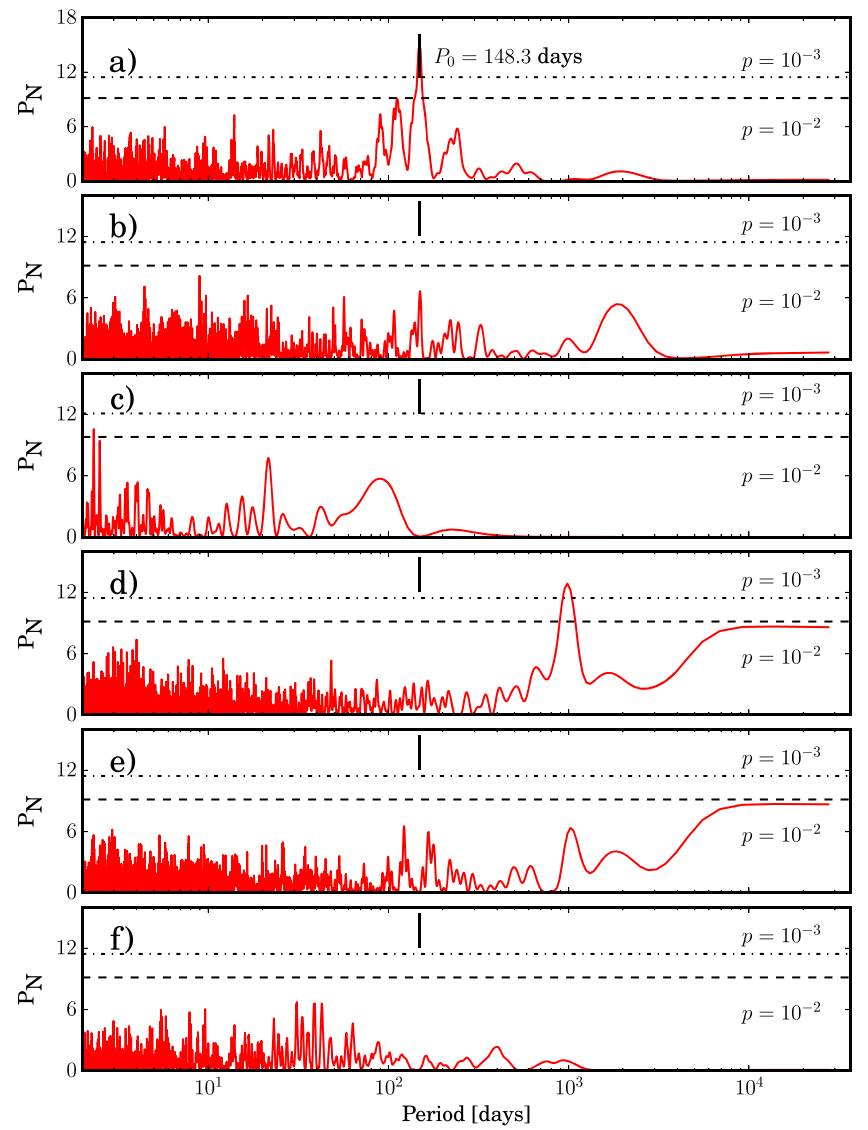

Figure 10. Lomb-Scargle periodograms for HD 216536. From top to bottom: HET RV data, BS, NSVS photometry (Woźniak et al. 2004), $I_{\mathrm{H} \alpha}, I_{\mathrm{Fe}}$, and RV post-fit residua.

\section{3. $H D 216536$}

The estimated projected rotation velocity of $v_{\text {rot }} \sin i_{\star}=2.6 \pm 0.5 \mathrm{~km} \mathrm{~s}^{-1}$ and the radius of $12.5 \pm 4.0 R_{\odot}$ of this star result in a maximum rotation period of $243 \pm 91$ days. HD 216536 had been observed by the NSVS (Woźniak et al. 2004) $5.6 \mathrm{yr}$ before the beginning of our measurements. Photometric observations of the star at 90 epochs between MJD 51304 and 51483 have been collected. These measurements are characterized by a mean brightness of $9.118 \mathrm{mag}$ and an rms scatter of $0.029 \mathrm{mag}$. As shown in the LS periodogram of Figure 10, no significant periodicity is present in the data. The observed photometric scatter could be explained in terms of a $f=2.9 \%$ spot rotating with the star. This would result in periodic 185 and $25 \mathrm{~m} \mathrm{~s}^{-1}$ variations in the RVs and BSs, respectively (Hatzes 2002). The fact that the predicted, spot-generated RV variations are similar in amplitude to those actually observed requires a careful analysis of the BS behavior.

The measured BSs exhibit variations that have a mean value of $14 \pm 22 \mathrm{~m} \mathrm{~s}^{-1}$, an amplitude of $106 \mathrm{~m} \mathrm{~s}^{-1}$, and a mean uncertainty of $14 \mathrm{~m} \mathrm{~s}^{-1}$. The Parsons correlation coefficient of the RV and BS variations amounts to 0.29 , which means that they are not likely to be correlated.

In addition, BS variations do not show any measurable periodicities. Consequently, the absence of any correlation between RVs and BSs and lack of periodic variations in both BS and existing photometry invalidates the spot hypothesis. 
Our measurements of $I_{\mathrm{H} \alpha}$ index for HD 216536 reveal a mean value of 0.0354 , and amplitude of variations of only $0.0021(6 \%)$. The $I_{\mathrm{Fe}}$ variations are even weaker-2.6\%. Both indices show a very similar pattern of LS and there is a significant signal at 984 days in the $I_{\mathrm{H} \alpha}$ data. The signal is well separated from the Keplerian period and the estimated maximum rotation period of $347 \pm 115$ days. The Parson's correlation between $I_{\mathrm{H} \alpha}$ and $I_{\mathrm{Fe}}, r=0.595$ is above critical $(r=0.376)$, which proves the instrumental nature of the signal. The RV variations are unrelated to that effect as the correlation coefficient between RV and $I_{\mathrm{H} \alpha}$ is only $r=0.055$.

We conclude that, according to the available evidence, the RV variations in HD 216536 cannot be related to rotationinduced stellar activity or stellar pulsations. Consequently, it is most logical to assume that these variations are due to the orbital motion of a planet-mass companion around the star. As for the other two stars, the observed BS variations must have another, unrelated source that is either random or not resolved in time with our observations.

\section{DISCUSSION. PLANETARY SYSTEMS OF EVOLVED STARS}

The three new stars with planets presented here provide a representative sample of the wealth of planetary system architectures observed in the evolved systems and illustrate how searches for planets around such stars can address various aspects of stellar and planetary system evolution.

By exploiting the approach of Ford \& Gregory (2007) and Johnson et al. (2011) in Keplerian orbit fitting we obtained independent estimates of stellar jitter, whose amount is consistent with the amplitudes of $p$-mode oscillations calculated from the scaling relations of Kjeldsen \& Bedding (1995). This proves that the L/M for the target stars and hence their positions on the HRD are correct.

HD 95127, a bright, $\log L / L_{\odot}=2.28 \pm 0.38$, solar-mass $M=1.2 \pm 0.22 M_{\odot}$ giant, with $R=20 \pm 9 R_{\odot}$ and $[\mathrm{Fe} /$ $\mathrm{H}]=-0.18 \pm 0.05$, hosts a $m \sin i=5.01_{-0.44}^{+0.61} M_{J}$ planet in a $1.28_{-0.01}^{+0.01} \mathrm{AU}, e=0.11_{-0.06}^{+0.15}$ orbit. The relatively low mass of the star and its expected large radius at the tip of the Red Giant Branch (RGB) set the current orbit of its planetary companion well within the engulfment zone (Villaver \& Livio 2009; Kunitomo et al. 2011; Villaver et al. 2014). This presents independent proof that the star is still evolving up the RGB.

HD 216536, a $M / M_{\odot}=1.36 \pm 0.38$ and $[\mathrm{Fe} / \mathrm{H}]=$ $-0.17 \pm 0.09$ star, within the parameter uncertainties, is also evolving up the RGB. Most likely this star is still before the helium ignition. It harbors one of the most close-in planets orbiting a giant, which supports our conclusion concerning its evolutionary stage. Given its $0.609_{-0.002}^{+0.002} \mathrm{AU}$ orbit, this $1.47_{-0.12}^{+0.20} M_{J}$ planet is one of the very few warm Jupiters around giants, prone to strong tidal interactions and most likely to be ingested before its host reaches the tip of the RGB (Villaver \& Livio 2009; Kunitomo et al. 2011; Villaver et al. 2014).

The position of $\mathrm{BD}+49828$ on the HRD suggests that this $\mathrm{M} / M_{\odot}=1.52 \pm 0.22$, slightly metal-deficient $([\mathrm{Fe} /$ $\mathrm{H}]=-0.19 \pm 0.06)$ star, is evolving up the RGB, possibly undergoing the first dredge-up. Its $m \sin i=1.6_{-0.2}^{+0.4} M_{J}$ companion, $4.2_{-0.2}^{+0.32} \mathrm{AU}$ away of the star, is one of the most distant planets orbiting giants detected so far. It is very likely that the planet will not be affected by stellar evolution and is similar to HD 4732 c (Sato et al. 2013) or HD 120084 b (Sato

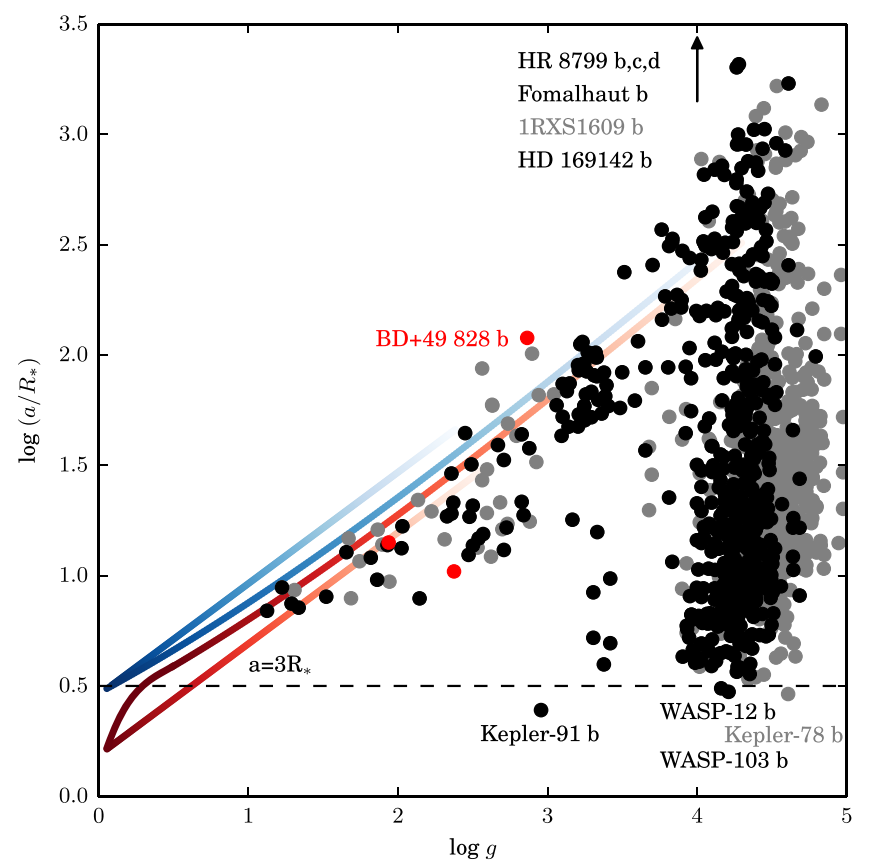

Figure 11. Orbital separation- $\log \left(a / R_{\star}\right)$ for planets orbiting hosts at various evolutionary stages- $\log (\mathrm{g})$, with all data from http://exoplanets.eu. Gray symbols_all planets; black symbols_-planets around 1-2 $M_{\odot}$ stars; red symbols - new planets presented here. Red and blue lines-minimum distance to avoid engulfment for a $1.5 M_{\odot}$ star and a $1 M_{J}$ planet system and minimum orbit above which a planet is not sensitive to tidal interaction with the stellar host from Villaver et al. (2014). See the text for discussion.

et al. 2013). Taking into account the star's uncertain luminosity estimate, the planet may also be located within the habitable zone as defined in Kopparapu (2013).

To put the three planetary candidates in a wider perspective, we plotted them as red circles in the $\log (\mathrm{g})$ versus $\log \left(a / R_{\star}\right)$ plane in Figure 11. Because the orbital radius, $a / R_{\star}$, is a major factor scaling the strength of tidal star-planet interactions, this plot may be regarded as an illustration of the tidal evolution of planetary systems. In the same Figure we have also placed all known exoplanets ${ }^{8}$ and KOIs that orbit stars with masses in the range of $1-2 M_{\odot}$ as black circles. Finally the total sample of planetary companions, for which at least semi-major axes, and stellar masses and radii, are available are shown as gray circles. For all stars, including the three presented here, uniform $\log g$ were calculated from stellar masses and radii adopted in the discovery papers. We note that for some planetary systems hosts the spectroscopic $\log g$ does not match those calculated from the stellar mass and radius. In particular this concerns 7 CMa (Wittenmyer et al. 2011), another long-period planet for which the host star $\log g_{M, R}=3.9$.

Also included in this Figure is the minimum distance to avoid engulfment for a $1 M_{J}$ planet orbiting a $1.5 M_{\odot}$ star under the Schröder \& Cuntz (2005) mass-loss prescription (red line) and the minimal orbit beyond which the planet is not affected by the tidal forces (blue line) from Villaver et al. (2014). The dashed line shows the $a / R_{\star}=3$ border inside of which tidal interactions are expected to lead quickly to ingestion. Note that the minimum distance to avoid engulfment for a $1 M_{J}$ planet orbiting a $1.5 M_{\odot}$ star from Villaver et al. (2014; red line) fits the general slope of decreasing $a / R$ with $\log g$ for known

8 All data from http://exoplanets.eu. 
planetary systems in Figure 11. In fact, up to a very late stellar evolution phase at RGB where $\log g<1$ and tidal ingestion is happening, this relation simply reflects the steady growth of a stellar radius.

An interesting feature of Figure 11 is that after the discoveries of HD 102956 b (Johnson et al. 2010), Kepler-56 b, c (Steffen et al. 2013), Kepler-391 b, c (Rowe et al. 2014), KOI-1299 b (Ciceri et al. 2014; Ortiz et al. 2014) and HIP $67851 \mathrm{~b}$ (Jones et al. 2014), the gap in orbital separations of planetary systems orbiting subgiants (here: $\log g=3.5 \pm 0.5$ ) pointed out in Nowak et al. (2013) no longer exists. A relatively low number of systems around those kinds of stars is obviously a consequence of a low population of that part of the HRD (see for example Figure 3.5.5 in ESA 1997).

The ingestion itself is supposed to be a quick process as clearly illustrated in Figure 11. Only three dwarfs (here: $\log g=4.5 \pm 0.5$; Wasp-12 b: Hebb et al. 2009; Kepler-78 b: Sanchis-Ojeda et al. 2013; Wasp-103 b: Gillon et al. 2014) out of 1037 or $\approx 0.3 \%$ are located in that range. The same is true for only one (Kepler-91 b) out of 44 or $\approx 2 \%$ giants (here: $\log g=2.5 \pm 0.5)$.

The most evolved planetary systems with $\log g<1.5$, very close to engulfment, are HD 220074 b, HD 208527 b (Lee et al. 2013), $\beta$ Cnc b (Lee et al. 2014), HD 96127 b (Gettel et al. 2012), and $\beta$ Umi b (Lee et al. 2014), all within $a / R_{\star}=9$. Interestingly enough, except for Kepler-91 b there is still no planet orbiting an evolved star with $\log g<3$ within $a / R_{\star}<\approx 7$ (the tightest orbit being HD $220074 \quad \mathrm{~b}$ at $\left.a / R_{\star}=6.93\right)$. This may suggest that tidal interactions between most extended evolved stars and their planets are more farreaching.

It is important to note that the part of Figure 11 occupied by bright giants (here: $\log g=1.5 \pm 0.5$ ) may be populated by both planets hosted by RG stars before the RGB tip and engulfment, and post-RGB-tip star planets. Although the latter planets have avoided tidal ingestion at the RGB tip, their orbits must have decayed (channel (2) in Villaver \& Livio 2009, see the discussion of their Figure 1), in some cases to well within $1 \mathrm{AU}$, and may subsequently be subjected to ingestion during their hosts Asymptotic Giant Branch evolution. Undoubtedly, all these stars deserve more detailed spectroscopic analysis to asses their evolutionary stages and hence the dynamical history of their planets in more detail.

All planets below the red line with at least one Jupiter mass are expected to be eventually ingested by their hosts before they reach the tip of the RGB. An obvious conotation from Figure 11 is that the vast majority of known planetary systems is located within the minimum distance to avoid engulfment (red line) and will not survive RGB evolution of their hosts, in agreement with Carlberg et al. (2009) and Nordhaus et al. (2010). Only very few known planetary systems contain planets in orbits, which are not affected by the tidal forces (above the blue line). This is 105 out of 1205 planets or $\approx 9 \%$ of planets in the http://exoplanet.eu catalog, for which at least semi-major axes, $M_{\star}$, and $R_{\star}$ are available. Most of the safe planets orbit dwarfs (77 out of 1037 or $\approx 7 \%$ ). A few such systems exist around subgiants (10 out of 99 or $\approx 10 \%$ ) with the most evolutionary advanced of them being HR $228 \mathrm{~b}$ and c (Sato et al. 2013). Only 5 (out of 44 or $\approx 11 \%$ ) of such systems are present around giants: HD 139357 b (Döllinger et al. 2009); $\nu$ Oph c and omi UMa b (Sato et al. 2012); HD 120084 b (Sato et al. 2013); and HD 14067 b (Wang et al. 2014). BD+49 $828 \mathrm{~b}$ presented here belongs to that group as well.

The obvious shortage of known safe planetary systems is most likely a consequence of current observational limitations. It illustrates the importance of long-term planet search projects such as, for example, PTPS, the Okayama Planet Search (Sato et al. 2005) or new projects like Friends of Hot Jupiters (Knutson et al. 2014). It also has serious consequences for the identification of asymmetric planetary nebular formation processes (Harpaz \& Soker 1994; Soker 1996; Livio \& Soker 2002).

The giant planets are formed outside the snow-line, at a distance of 6-12 AU in the case of 1-2 $M_{\odot}$ stars (Kennedy \& Kenyon 2008). They migrate inward (Armitage 2007) in the protoplanetary disks through type II migration but other migration scenarios (planet-planet scattering (Rasio \& Ford 1996; Ford et al. 2001) or Kozai migration (Kozai 1962; Wu \& Murray 2003)) are also possible. Such planets may actually become dominant when the protoplanetary disk is dispersed. The efficiency of the migration process, and especially a relative efficiency of various mechanisms, is basically unknown due to the lack of known long-period planets. We note the importance of Rositer-McLaughlin effect studies in that context (Simpson et al. 2010).

An inventory of planetary system at various evolutionary stages of their hosts is presented in Table 6, in which four sets of data are shown: all stars for which all parameters listed in Table 6 are available, and a subset of these stars limited to 1-2 $M_{\odot}$, as well as subsets of those two sets with the RV detected planets only. In the case of dwarfs, we see the impact of shortperiod transit planets as a huge variation between the mean value and the median of minimum planetary semi-major axis, hence the need for a "RV-limited" subsamples.

Several interesting features of the known sample of exoplanets emerge. In RV-limited subsamples not much variation in semi-major amplitude or eccentricity is seen between planetary systems at various evolutionary stages of their hosts. Both a and e vary by about $1 \sigma$ between dwarfs and bright giants, ruling out tidal circularization during the stellar evolution.

Another important general feature of the known planet sample is the steady decrease of metallicity as we move from dwarfs to several billion year older giants. That reflects the changes in stellar populations with which we are dealing, with varying star formation rate (Tremblay et al. 2014) possibly reflected in the planet formation rate (Boss 2002; Johnson \& Li 2012, 2013; Hasegawa \& Hirashita 2014).

As we move from dwarfs to more evolved hosts we deal with more massive stars on average as well. An average dwarf with a planetary system is a $\approx 1 M_{\odot}$ (F or G spectral type) star while an average subgiant is already $\mathrm{a} \approx 1.5 M_{\odot}$ (MS spectral type AF) star, and a giant (MS A-type star) may be almost twice as massive as a dwarf (5-10 $\sigma$ difference). Stellar mass is not expected to increase during MS and RSG evolution and it is clear that we must be facing a strong selection effect here. Even in a narrow 1-2 $M_{\odot}$ mass range we still see more massive, evolved stars. On one hand, masses of single evolved stars are not easy to estimate since they may be simply overestimated (Lloyd 2011, 2013; Schlaufman \& Winn 2013) which would be the easiest explanation of the suspicious lack of solar-mass evolved planetary systems hosts. On the other hand, taking into account that a solar-mass star is expected to lose $\sim 0.2-0.4 M_{\odot}$ 
Table 6

Basic Parameters of Planets Around Stars at Various Stages of Stellar Evolution (from http://exoplanets.eu)

\begin{tabular}{|c|c|c|c|c|c|c|c|c|c|}
\hline Parameter & $M_{\star}$ Range & Dwarfs & & Subgiant & & Giants & & Bright Giants & $\cdots$ \\
\hline & & $\begin{array}{l}\text { mean } \\
\text { median }\end{array}$ & $\sigma$ & $\begin{array}{l}\text { mean } \\
\text { median }\end{array}$ & $\sigma$ & $\begin{array}{l}\text { mean } \\
\text { median }\end{array}$ & $\sigma$ & $\begin{array}{l}\text { mean } \\
\text { median }\end{array}$ & $\sigma$ \\
\hline \multirow[t]{4}{*}{$\mathrm{N}_{\text {planet }}$} & $1-2 M_{\odot}$ & 267 & $\cdots$ & 73 & $\cdots$ & 23 & $\cdots$ & 9 & $\cdots$ \\
\hline & all & 458 & $\ldots$ & 77 & $\ldots$ & 41 & $\ldots$ & 14 & $\ldots$ \\
\hline & $1-2 M_{\odot}(\mathrm{RV})$ & 138 & $\ldots$ & 60 & $\ldots$ & 22 & $\cdots$ & 9 & $\cdots$ \\
\hline & all(RV) & 236 & $\cdots$ & 64 & $\cdots$ & 40 & $\cdots$ & 14 & $\cdots$ \\
\hline \multirow[t]{8}{*}{$\log (g)$} & all & 4.378 & 0.009 & 3.540 & 0.037 & 2.574 & 0.039 & 1.594 & 0.077 \\
\hline & $\ldots$ & 4.377 & $\ldots$ & 3.394 & $\ldots$ & 2.594 & $\ldots$ & 1.663 & $\ldots$ \\
\hline & $1-2 M_{\odot}$ & 4.285 & 0.008 & 3.533 & 0.037 & 2.533 & 0.054 & 1.529 & 0.100 \\
\hline & $\ldots$ & 4.282 & & 3.386 & $\ldots$ & 2.501 & $\ldots$ & 1.521 & $\cdots$ \\
\hline & $\operatorname{all}(\mathrm{RV})$ & 4.392 & 0.013 & 3.485 & 0.038 & 2.564 & 0.038 & 1.594 & 0.077 \\
\hline & $\ldots$ & 4.392 & $\ldots$ & 3.369 & $\ldots$ & 2.582 & $\ldots$ & 1.663 & $\ldots$ \\
\hline & $1-2 M_{\odot}(\mathrm{RV})$ & 4.296 & 0.012 & 3.473 & 0.038 & 2.514 & 0.053 & 1.529 & 0.100 \\
\hline & $\ldots$ & 4.300 & $\cdots$ & 3.350 & $\cdots$ & 2.501 & $\cdots$ & 1.521 & $\cdots$ \\
\hline \multirow[t]{8}{*}{$M_{\star} / M_{\odot}$} & all & 1.039 & 0.011 & 1.428 & 0.027 & 1.870 & 0.090 & 1.464 & 0.120 \\
\hline & $\ldots$ & 1.031 & $\ldots$ & 1.450 & $\ldots$ & 1.900 & $\ldots$ & 1.395 & $\ldots$ \\
\hline & $1-2 M_{\odot}$ & 1.179 & 0.009 & 1.453 & 0.025 & 1.499 & 0.060 & 1.457 & 0.084 \\
\hline & $\ldots$ & 1.140 & & 1.470 & $\ldots$ & 1.500 & $\ldots$ & 1.400 & $\ldots$ \\
\hline & $\operatorname{all}(\mathrm{RV})$ & 0.997 & 0.015 & 1.446 & 0.031 & 1.885 & 0.091 & 1.464 & 0.120 \\
\hline & $\cdots$ & 1.030 & $\cdots$ & 1.475 & $\ldots$ & 1.900 & $\ldots$ & 1.395 & $\cdots$ \\
\hline & $1-2 M_{\odot}(\mathrm{RV})$ & 1.140 & 0.010 & 1.478 & 0.028 & 1.507 & 0.062 & 1.457 & 0.084 \\
\hline & $\cdots$ & 1.100 & $\cdots$ & 1.480 & $\cdots$ & 1.500 & $\cdots$ & 1.400 & $\cdots$ \\
\hline \multirow[t]{8}{*}[\mathrm{Fe}/\mathrm{H}]{} & all & 0.071 & 0.010 & 0.086 & 0.022 & -0.032 & 0.032 & -0.247 & 0.058 \\
\hline & $\ldots$ & 0.090 & $\ldots$ & 0.120 & $\ldots$ & -0.052 & $\ldots$ & -0.255 & $\ldots$ \\
\hline & $1-2 M_{\odot}$ & 0.114 & 0.011 & 0.099 & 0.020 & -0.012 & 0.045 & -0.222 & 0.055 \\
\hline & $\ldots$ & 0.140 & $\ldots$ & 0.140 & $\ldots$ & -0.030 & $\ldots$ & -0.260 & $\ldots$ \\
\hline & all(RV) & 0.102 & 0.014 & 0.093 & 0.026 & -0.036 & 0.033 & -0.247 & 0.058 \\
\hline & $\ldots$ & 0.140 & $\ldots$ & 0.140 & $\ldots$ & -0.056 & $\ldots$ & -0.255 & $\ldots$ \\
\hline & $1-2 M_{\odot}(\mathrm{RV})$ & 0.178 & 0.012 & 0.109 & 0.023 & -0.018 & 0.046 & -0.222 & 0.055 \\
\hline & $\cdots$ & 0.210 & $\cdots$ & 0.140 & $\cdots$ & -0.060 & $\cdots$ & -0.260 & $\cdots$ \\
\hline \multirow[t]{8}{*}{$\mathrm{m}_{P} \operatorname{sini} / \mathrm{m}_{J}$} & all & 2.046 & 0.147 & 2.702 & 0.399 & 6.391 & 0.987 & 7.798 & 1.186 \\
\hline & $\cdots$ & 0.955 & $\cdots$ & 1.800 & $\cdots$ & 4.500 & $\cdots$ & 6.300 & $\cdots$ \\
\hline & $1-2 M_{\odot}$ & 2.459 & 0.212 & 2.705 & 0.416 & 4.557 & 0.592 & 7.688 & 0.854 \\
\hline & $\cdots$ & 1.308 & & 1.800 & $\ldots$ & 3.200 & $\ldots$ & 7.800 & $\cdots$ \\
\hline & $\operatorname{all}(\mathrm{RV})$ & 2.250 & 0.200 & 2.956 & 0.467 & 6.533 & 1.001 & 7.798 & 1.186 \\
\hline & $\ldots$ & 1.135 & $\ldots$ & 1.850 & $\ldots$ & 4.900 & $\ldots$ & 6.300 & $\ldots$ \\
\hline & $1-2 M_{\odot}(\mathrm{RV})$ & 2.532 & 0.256 & 2.977 & 0.492 & 4.731 & 0.592 & 7.688 & 0.854 \\
\hline & $\ldots$ & 1.658 & $\ldots$ & 1.850 & $\ldots$ & 3.260 & $\ldots$ & 7.800 & $\cdots$ \\
\hline \multirow[t]{8}{*}{$\mathrm{e}$} & all & 0.162 & 0.010 & 0.185 & 0.018 & 0.187 & 0.031 & 0.220 & 0.048 \\
\hline & $\cdots$ & 0.072 & $\cdots$ & 0.160 & $\cdots$ & 0.110 & $\cdots$ & 0.167 & $\cdots$ \\
\hline & $1-2 M_{\odot}$ & 0.165 & 0.013 & 0.178 & 0.018 & 0.177 & 0.045 & 0.207 & 0.068 \\
\hline & $\ldots$ & 0.070 & $\cdots$ & 0.157 & $\cdots$ & 0.090 & $\ldots$ & 0.140 & $\cdots$ \\
\hline & $\operatorname{all}(\mathrm{RV})$ & 0.244 & 0.014 & 0.212 & 0.019 & 0.190 & 0.032 & 0.220 & 0.048 \\
\hline & $\cdots$ & 0.201 & $\cdots$ & 0.185 & $\cdots$ & 0.111 & $\cdots$ & 0.167 & $\cdots$ \\
\hline & $1-2 M_{\odot}(\mathrm{RV})$ & 0.260 & 0.018 & 0.205 & 0.019 & 0.182 & 0.047 & 0.207 & 0.068 \\
\hline & $\ldots$ & 0.225 & $\ldots$ & 0.179 & $\ldots$ & 0.095 & $\ldots$ & 0.140 & $\cdots$ \\
\hline \multirow[t]{8}{*}{$\mathrm{a} / \mathrm{AU}$} & all & 1.116 & 0.193 & 1.331 & 0.111 & 1.476 & 0.172 & 1.487 & 0.085 \\
\hline & $\cdots$ & 0.094 & $\cdots$ & 1.310 & $\cdots$ & 1.200 & $\cdots$ & 1.470 & $\cdots$ \\
\hline & $1-2 M_{\odot}$ & 1.442 & 0.324 & 1.358 & 0.115 & 1.119 & 0.113 & 1.517 & 0.119 \\
\hline & $\ldots$ & 0.095 & $\ldots$ & 1.310 & $\ldots$ & 1.160 & $\ldots$ & 1.540 & $\ldots$ \\
\hline & all(RV) & 1.506 & 0.108 & 1.568 & 0.112 & 1.511 & 0.173 & 1.487 & 0.085 \\
\hline & $\cdots$ & 0.983 & $\cdots$ & 1.450 & $\cdots$ & 1.235 & $\cdots$ & 1.470 & $\cdots$ \\
\hline & $1-2 M_{\odot}(\mathrm{RV})$ & 1.725 & 0.149 & 1.617 & 0.115 & 1.166 & 0.107 & 1.517 & 0.118 \\
\hline & $\ldots$ & 1.188 & $\ldots$ & 1.515 & $\ldots$ & 1.180 & $\ldots$ & 1.540 & $\ldots$ \\
\hline
\end{tabular}

Note. Mean and median values as well as dispersion are presented for every set of data. See the text for details. 
Table 7

Parsons Correlation Coefficients between Stellar Host Mass and Total Mass of Planetary Systems

\begin{tabular}{|c|c|c|c|c|c|c|c|c|}
\hline \multirow[t]{2}{*}{ Group } & \multicolumn{4}{|c|}{$\mathrm{M}_{\mathrm{PS}}$ Versus $\mathrm{M}_{*}$} & \multicolumn{4}{|c|}{$N_{\text {systems }}$} \\
\hline & $1-2 M_{\odot}$ & all & $1-2 M_{\odot}(\mathrm{RV})$ & all (RV) & $1-2 M_{\odot}$ & all & $1-2 M_{\odot}(\mathrm{RV})$ & all (RV) \\
\hline \multirow[t]{2}{*}{$\overline{\text { Dwarfs }}$} & 0.222 & 0.132 & 0.194 & 0.234 & 256 & 432 & 100 & 172 \\
\hline & 0.137 & 0.172 & 0.202 & 0.223 & 220 & 367 & 99 & 167 \\
\hline Subgiants & 0.119 & 0.113 & 0.095 & 0.099 & 64 & 68 & 53 & 57 \\
\hline \multirow[t]{2}{*}{ Giants } & 0.101 & 0.409 & 0.057 & 0.400 & 21 & 40 & 20 & 39 \\
\hline & 0.182 & 0.480 & 0.144 & 0.469 & 20 & 37 & 19 & 36 \\
\hline \multirow[t]{2}{*}{ All } & 0.204 & 0.234 & 0.164 & 0.402 & 354 & $567^{\mathrm{a}}$ & 184 & 286 \\
\hline & 0.181 & 0.380 & 0.172 & 0.407 & 313 & 486 & 180 & 274 \\
\hline
\end{tabular}

Note. Upper values: all systems for which at least masses are available; lower values: systems with all data listed in Table 6 available. All correlation coefficients are smaller than the respective critical values.

${ }^{\mathrm{a}}$ Including 6 hosts with $\log (\mathrm{g})>5$.

in the RG stage (Christy 1966; Fusi Pecci et al. 1993; Lebzelter \& Wood 2005; Kalirai et al. 2007; McDonald et al. 2011) and remembering that the fraction of RGB mass loss increases with stellar mass (Kalirai et al. 2008), we see that the known evolved planetary system hosts might have been originally $\approx 2-3 M_{\odot}$ MS stars, at the low-intermediate-mass border or above. This points to their very uncertain, possibly quite complicated evolutionary history.

The planetary mass increase for more evolved hosts seems to be the most prominent feature present in Table 6. An average giant hosts a companion about twice as massive than in the case of a dwarf, and a bright giant's companion is three times more massive, on average. Certainly, that is not an observational bias caused by RV jitter present in giants as companions below Jupiter mass can be easily detected around those stars (for instance BD+48738 b, Gettel et al. 2012). Assuming an average value of $\sin i$, companions to bright giants are, on average, at the brown dwarf-planet borderline (see also Mitchell et al. 2013). It is rather obvious that such an amount of mass cannot be accreted by a giant planet during its host's RGB evolution (Duncan \& Lissauer 1998).

We note, however, a lack of correlation between current host mass and its observed planetary system mass (Table 7). It does not necessarily mean that there is no relation between hosts and their planetary system masses, as postulated in Lovis \& Mayor (2007). We may speculate that, to show such a relation, one should use initial masses of the planetary system hosts, instead of the current ones, corrected for mass loss over their entire evolution, and account for the planetary system mass loss through planet ingestion or ejection. If we assume, for simplicity, that an average subgiant from Table 6 (RV-limited, complete sample mean values of mass) lost $5 \%$ of its initial mass, whereas a giant and a bright giant would lose $15 \%$ and $35 \%$, respectively, we obtain a perfect host mass-planetary mass relation with a correlation coefficient $r=0.95$, which is statistically significant at the $99 \%$ confidence level. This result is promising enough to justify a more detailed study.

We thank the HET resident astronomers and telescope operators for continuous support. A.N., M.o.A., B.D.-S., M.i.A. and K.K. are currently supported by NCN grant 2012/ 07/B/ST9/04415. M.o.A. also acknowledges the "Mobility + III" fellowship from the Polish Ministry of Science and Higher Education. A.W. was supported by the NASA grant
NNX09AB36G. G.M. acknowledges the financial support from the Polish Ministry of Science and Higher Education through the Iuventus Plus grant IP2011 031971. The HET is a joint project of the University of Texas at Austin, the Pennsylvania State University, Stanford University, LudwigMaximilians-Universität München, and Georg-August-Universität Göttingen. The HET is named in honor of its principal benefactors, William P. Hobby and Robert E. Eberly. The Center for Exoplanets and Habitable Worlds is supported by the Pennsylvania State University and the Eberly College of Science. This research has made extensive use of the SIMBAD database, operated at CDS (Strasbourg, France) and NASA's Astrophysics Data System Bibliographic Services.

\section{REFERENCES}

Adamów, M., Niedzielski, A., Villaver, E., Wolszczan, A., \& Nowak, G. 2014, A\&A, 569, A55

Armitage, P. J. 2007, ApJ, 665, 1381

Baglin, A., Auvergne, M., Barge, P., et al. 2006, ESA Special Publication, 1306, 33

Barclay, T., Endl, M., Huber, D., et al. 2014, arXiv:1408.3149

Bedding, T. R., Huber, D., Stello, D., et al. 2010, ApJL, 713, L176

Bertelli, G., Girardi, L., Marigo, P., \& Nasi, E. 2008, A\&A, 484, 815

Bressan, A., Marigo, P., Girardi, L., et al. 2012, MNRAS, 427, 127

Boss, A. P. 2002, ApJL, 567, L149

Borucki, W. J., Koch, D. G., Basri, G., et al. 2011, ApJ, 736, 19

Bowler, B. P., Johnson, J. A., Marcy, G. W., et al. 2010, ApJ, 709, 396

Butler, R. P., Marcy, G. W., Williams, E., et al. 1996, PASP, 108, 500

Butler, R. P., Tinney, C. G., Marcy, G. W., et al. 2001, ApJ, 555, 410

Butters, O. W., West, R. G., Anderson, D. R., et al. 2010, A\&A, 520, L10

Campbell, B., Walker, G. A. H., \& Yang, S. 1988, ApJ, 331, 902

Cannon, A. J., \& Pickering, E. C. 1925, Annals of the Astronomical Observatory of Harvard College (Cambridge, MA: Astronomical Observatory of Harvard College)

Carlberg, J. K., Majewski, S. R., \& Arras, P. 2009, ApJ, 700, 832

Charbonneau, P. 1995, ApJS, 101, 309

Choi, K. H. 1980, PhD thesis, Pennsylvania University

Ciceri, S., Lillo-Box, J., Southworth, J., et al. 2014, arXiv:1410.2999

Choi, K. H., \& Vila, S. C. 1981, Ap\&SS, 77, 319

Christy, R. F. 1966, ApJ, 144, 108

Cochran, W. D., Endl, M., McArthur, B., et al. 2004, ApJL, 611, L133

Counselman, C. C., III 1973, ApJ, 180, 307

Dieckvoss, W., \& Heckmann, O. 1997, yCat, 1061, 0

Döllinger, M. P., Hatzes, A. P., Pasquini, L., et al. 2009, A\&A, 499, 935

Duncan, M. J., \& Lissauer, J. J. 1998, Icar, 134, 303

ESA 1997, ESA Special Publication 1200, The HIPPARCOS and TYCHO Catalogues

Frink, S., Mitchell, D. S., Quirrenbach, A., et al. 2002, ApJ, 576, 478

Ford, E. B., Havlickova, M., \& Rasio, F. A. 2001, Icar, 150, 303 
Ford, E. B., \& Gregory, P. C. 2007, Statistical Challenges in Modern Astronomy IV, 371, 189

Fusi Pecci, F., Ferraro, F. R., Bellazzini, M., et al. 1993, AJ, 105, 1145

Gettel, S., Wolszczan, A., Niedzielski, A., et al. 2012, ApJ, 745, 28

Gillon, M., Anderson, D. R., Collier-Cameron, A., et al. 2014, A\&A, 562, LL3

Gilliland, R. L., Brown, T. M., Christensen-Dalsgaard, J., et al. 2010, PASP, 122,131

Gomes da Silva, J., Santos, N. C., Bonfils, X., et al. 2012, A\&A, 541, A9

Goździewski, K., Konacki, M., \& Maciejewski, A. J. 2003, ApJ, 594, 1019

Goździewski, K., \& Migaszewski, C. 2006, A\&A, 449, 1219

Goździewski, K., Maciejewski, A. J., \& Migaszewski, C. 2007, ApJ, 657, 546

Harpaz, A., \& Soker, N. 1994, MNRAS, 270, 734

Hatzes, A. P. 2002, AN, 323, 392

Hatzes, A. P., \& Cochran, W. D. 1993, ApJ, 413, 339

Hatzes, A. P., \& Cochran, W. D. 1994, ApJ, 432, 763

Hatzes, A. P., Cochran, W. D., Endl, M., et al. 2003, ApJ, 599, 1383

Hatzes, A. P., Cochran, W. D., Endl, M., et al. 2006, A\&A, 457, 335

Hebb, L., Collier-Cameron, A., Loeillet, B., et al. 2009, ApJ, 693, 1920

Hasegawa, Y., \& Hirashita, H. 2014, ApJ, 788, 62

Hekker, S., Gilliland, R. L., Elsworth, Y., et al. 2011, MNRAS, 414, 2594

Høg, E., Fabricius, C., MaKarov, V. V., et al. 2000, A\&A, 355, L27

Houk, N. 1963, AJ, 68, 253

Jackson, B., Barnes, R., \& Greenberg, R. 2009, ApJ, 698, 1357

Johnson, J. A., Fischer, D. A., Marcy, G. W., et al. 2007, ApJ, 665, 785

Johnson, J. A., Bowler, B. P., Howard, A. W., et al. 2010, ApJL, 721, L153

Johnson, J. A., Clanton, C., Howard, A. W., et al. 2011, ApJS, 197, 26

Jørgensen, B. R., \& Lindegren, L. 2005, A\&A, 436, 127

Johnson, J. L., \& Li, H. 2013, MNRAS, 431, 972

Johnson, J. L., \& Li, H. 2012, ApJ, 751, 81

Jones, M. I., Jenkins, J. S., Rojo, P., Melo, C. H. F., \& Bluhm, P. 2014, arXiv: 1409.7429

Kalirai, J. S., Bergeron, P., Hansen, B. M. S., et al. 2007, ApJ, 671, 748

Kalirai, J. S., Hansen, B. M. S., Kelson, D. D., et al. 2008, ApJ, 676, 594

Knutson, H. A., Fulton, B. J., Montet, B. T., et al. 2014, ApJ, 785, 126

Kozai, Y. 1962, AJ, 67, 591

Kallinger, T., Mosser, B., Hekker, S., et al. 2010, A\&A, 522, A1

Kennedy, G. M., \& Kenyon, S. J. 2008, ApJ, 673, 502

Kjeldsen, H., \& Bedding, T. R. 1995, A\&A, 293, 87

Kopal, Z. 1972, Ap\&SS, 16, 3

Kopparapu, R. K., et al. 2013, ApJ, 765, 131

Kuerster, M., Schmitt, J. H. M. M., Cutispoto, G., \& Dennerl, K. 1997, A\&A, 320,831

Kunitomo, M., Ikoma, M., Sato, B., Katsuta, Y., \& Ida, S. 2011, ApJ, 737, 66

Lillo-Box, J., Barrado, D., Moya, A., et al. 2014, A\&A, 562, A109

Lebzelter, T., \& Wood, P. R. 2005, A\&A, 441, 1117

Lee, B.-C., Han, I., Park, M.-G., et al. 2014, A\&A, 566, A67

Lee, B.-C., Han, I., \& Park, M.-G. 2013, A\&A, 549, A2

Liu, Y.-J., Sato, B., Zhao, G., et al. 2008, ApJ, 672, 553

Livio, M. 1982, A\&A, 112, 190

Livio, M., \& Soker, N. 1983, A\&A, 125, L12

Livio, M., \& Soker, N. 1984, MNRAS, 208, 763

Livio, M., \& Soker, N. 2002, ApJL, 571, L161

Lomb, N. R. 1976, Ap\&SS, 39, 447

Lovis, C., \& Mayor, M. 2007, A\&A, 472, 657

Maciejewski, G., Niedzielski, A., Wolszczan, A., et al. 2013, AJ, 146, 147

Marcy, G. W., \& Butler, R. P. 1992, PASP, 104, 270

Marcy, G. W., Butler, R. P., Vogt, S. S., et al. 2005, ApJ, 619, 570

Markwardt, C. B. 2009, ADASS, 411, 251

Mitchell, D. S., Reffert, S., Trifonov, T., Quirrenbach, A., \& Fischer, D. A. 2013, A\&A, 555, A87

Mortier, A., Santos, N. C., Sousa, S. G., et al. 2013, A\&A, 557, A70

Mitchell, D. S., Reffert, S., Trifonov, T., Quirrenbach, A., \& Fischer, D. A. 2013, A\&A, 555, A87

McDonald, I., Johnson, C. I., \& Zijlstra, A. A. 2011, MNRAS, 416, L6

Murdoch, K. A., Hearnshaw, J. B., \& Clark, M. 1993, ApJ, 413, 349

Nicholls, C. P., Wood, P. R., Cioni, M.-R. L., \& Soszyński, I. 2009, MNRAS, 399, 2063

Niedzielski, A., Nowak, G., Adamów, M., \& Wolszczan, A. 2009, ApJ, 707, 768

Niedzielski, A., Wolszczan, A., Adamów, M., et al. 2013, MmSAI, 84, 1035

Niedzielski, A., Konacki, M., Wolszczan, A., et al. 2007, ApJ, 669, 1354
Niedzielski, A., \& Wolszczan, A. 2008, in IAU Symp. vol. 249, ed. Y. S. Sun, S. Ferraz-Mello, \& J. L. Zhou (Cambridge: Cambridge Univ. Press), 43

Nordhaus, J., Spiegel, D. S., Ibgui, L., Goodman, J., \& Burrows, A. 2010, MNRAS, 408, 631

Nordhaus, J., \& Spiegel, D. S. 2013, MNRAS, 432, 500

Nowak, G. 2012, PhD thesis, Nicolaus Copernicus Univ., Toruń, Poland

Nowak, G., Niedzielski, A., Wolszczan, A., Adamów, M., \& Maciejewski, G. 2013, ApJ, 770, 53

Ortiz, M., Gandolfi, D., Reffert, S., et al. 2014, arXiv:1410.3000

Payne-Gaposchkin, C. 1954, AnHar, 113, 189

Pepe, F., Mayor, M., Galland, F., et al. 2002, A\&A, 388, 632

Pollacco, D. L., Skillen, I., Collier Cameron, A., et al. 2006, PASP, 118, 1407

Press, W. H., Teukolsky, S. A., Vetterling, W. T., \& Flannery, B. P. 1992, Numerical Recipes in FORTRAN. The Art of Scientific Computing (2nd ed.; Cambridge: Cambridge Univ. Press)

Queloz, D. 1995, in IAU Symp. 167, New Developments in Array Technology and Applications, ed. A. G. D. Philip, K. Janes, \& A. R. Upgren (Cambridge: Cambridge Univ. Press), 221

Ramsey, L. W., Adams, M. T., Barnes, T. G., et al. 1998, Proc. SPIE, 3352, 34

Rasio, F. A., Tout, C. A., Lubow, S. H., \& Livio, M. 1996, ApJ, 470, 1187

de Ridder, J., Barban, C., Baudin, F., et al. 2009, Natur, 459, 398

Robertson, P., Endl, M., Cochran, W. D., \& Dodson-Robinson, S. E. 2013, ApJ, 764, 3

Rasio, F. A., \& Ford, E. B. 1996, Sci, 274, 954

Rowe, J. F., Bryson, S. T., Marcy, G. W., et al. 2014, ApJ, 784, 45

Rybicki, K. R., \& Denis, C. 2001, Icar, 151, 130

Sanchis-Ojeda, R., Rappaport, S., Winn, J. N., et al. 2013, ApJ, 774, 54

Simpson, E. K., Pollacco, D., Hébrard, G., et al. 2010, MNRAS, 405, 1867

Soker, N. 1996, ApJ, 468, 774

Steffen, J. H., Fabrycky, D. C., Agol, E., et al. 2013, MNRAS, 428, 1077

Sato, B., Izumiura, H., Toyota, E., et al. 2007, ApJ, 661, 527

Sato, B., Izumiura, H., Toyota, E., et al. 2008, PASJ, 60, 539

Sato, B., Kambe, E., Takeda, Y., et al. 2005, PASJ, 57, 97

Sato, B., Omiya, M., Harakawa, H., et al. 2012, PASJ, 64, 135

Sato, B., Omiya, M., Liu, Y., et al. 2010, PASJ, 62, 1063

Sato, B., Omiya, M., Wittenmyer, R. A., et al. 2013, ApJ, 762, 9

Scargle, J. D. 1982, ApJ, 263, 835

Schröder, K.-P., \& Connon Smith, R. 2008, MNRAS, 386, 155

Siess, L., \& Livio, M. 1999a, MNRAS, 308, 1133

Siess, L., \& Livio, M. 1999b, MNRAS, 304, 925

Schröder, K.-P., \& Cuntz, M. 2005, ApJL, 630, L73

Schwamb, M. E., Orosz, J. A., Carter, J. A., et al. 2013, ApJ, 768, 127

Shetrone, M., Cornell, M. E., Fowler, J. R., et al. 2007, PASP, 119, 556

da Silva, L., Girardi, L., Pasquini, L., et al. 2006, A\&A, 458, 609

Stumpff, P. 1980, A\&AS, 41, 1

Takeda, Y., Ohkubo, M., Sato, B., Kambe, E., \& Sadakane, K. 2005a, PASJ, 57, 27

Takeda, Y., Sato, B., Kambe, E., et al. 2005b, PASJ, 57, 109

Tremblay, P.-E., Kalirai, J. S., Soderblom, D. R., Cignoni, M., \& Cummings, J. 2014, ApJ, 791, 92

Tull, R. G. 1998, Proc. SPIE, 3355, 387

van Leeuwen, F. 2007, A\&A, 474, 653

Valenti, J. A., \& Piskunov, N. 1996, A\&AS, 118, 595

Veras, D., Evans, N. W., Wyatt, M. C., \& Tout, C. A. 2014, MNRAS, 437, 1127

Vila, S. C. 1984, EM\&P, 31, 313

Villaver, E., \& Livio, M. 2007, ApJ, 661, 1192

Villaver, E., \& Livio, M. 2009, ApJL, 705, L81

Villaver, E., Livio, M., Mustill, A. J., \& Siess, L. 2014, ApJ, 794, 3

Vogt, S. S., Marcy, G. W., Butler, R. P., \& Apps, K. 2000, ApJ, 536, 902

Walker, G. A. H., Yang, S., Campbell, B., \& Irwin, A. W. 1989, ApJL, 343, L21

Wang, L., Sato, B., Omiya, M., et al. 2014, arXiv:1409.6081

Woźniak, P. R., Vestrand, W. T., Akerlof, C. W., et al. 2004, AJ, 127, 2436

Wright, J. T., Marcy, G. W., Fischer, D. A., et al. 2007, ApJ, 657, 533

Wright, J. T., \& Howard, A. W. 2009, ApJS, 182, 205

Wu, Y., \& Murray, N. 2003, ApJ, 589, 605

Wittenmyer, R. A., Endl, M., Wang, L., et al. 2011, ApJ, 743, 184

Zahn, J.-P. 1977, A\&A, 57, 383

Zieliński, P., Niedzielski, A., Wolszczan, A., Adamów, M., \& Nowak, G. 2012, A\&A, 547, A91 